\title{
Spatial patterns of tumour growth impact clonal diversification: computational modelling and evidence in the TRACERx Renal study
}

\section{Xiao Fu}

The Francis Crick Institute https://orcid.org/0000-0002-8611-891X

\section{Yue Zhao}

The Francis Crick Institute

Jose Lopez

Cruces University Hospital

\section{Andrew Rowan}

The Francis Crick Institute

\section{Lewis Au}

The Francis Crick Institute

\section{Annika Fendler}

The Francis Crick Institute

\section{Steve Hazell}

The Royal Marsden NHS Foundation Trust

\section{Hang Xu}

Stanford Cancer Institute https://orcid.org/0000-0002-5847-655X

\section{Stuart Horswell}

The Francis Crick Institute

\section{Scott Shepherd}

The Francis Crick Institute https://orcid.org/0000-0001-6208-173X

\section{Lavinia Spain}

The Royal Marsden Hospital NHS Foundation Trust

Fiona Byrne

The Francis Crick Institute https://orcid.org/0000-0003-3800-0500

\section{Gordon Stamp}

The Francis Crick Institute

\section{Tim O'Brien}

Guy's \& St Thomas' NHS Foundation Trust

\section{David Nicol}

The Royal Marsden NHS Foundation Trust

\section{Marcellus Augustine}


The Francis Crick Institute

\section{Ashish Chandra}

Guy's \& St Thomas' NHS Foundation Trust

\section{Sarah Rudman}

Guy's \& St. Thomas' NHS Foundation Trust

\section{Antonia Toncheva}

Guy's and St. Thomas' NHS Foundation Trust

\section{Andrew Furness}

The Francis Crick Institute

\section{Lisa Pickering}

The Royal Marsden Hospital NHS Foundation Trust https://orcid.org/0000-0002-7579-340X

\section{Matthew Orton}

Institute of Cancer Research

\section{Simon Doran}

Institute of Cancer Research https://orcid.org/0000-0001-8569-9188

\section{Dow-Mu Koh}

Institute of Cancer Research

\section{Christina Messiou}

Institute of Cancer Research

\section{Derfel ap Dafydd}

Institute of Cancer Research

\section{Santosh Kumar}

Institute of Cancer Research

James Larkin

The Royal Marsden Hospital NHS Foundation Trust

\section{Charles Swanton}

The Francis Crick Institute https://orcid.org/0000-0002-4299-3018

\section{Erik Sahai}

The Francis Crick Institute

\section{Kevin Litchfield}

The Francis Crick Institute

\section{Samra Turajlic}

Francis Crick Institute https://orcid.org/0000-0001-8846-136X

\section{Paul Bates ( $\nabla$ paul.bates@crick.ac.uk)}

The Francis Crick Institute https://orcid.org/0000-0003-0621-0925

\section{Article}


Keywords: Computational modelling, Cellular Automaton, Clear cell renal cell carcinoma, Spatial growth 3 patterns, Microdiversity, Parallel evolution, Time-course analysis

Posted Date: February 19th, 2021

DOI: https://doi.org/10.21203/rs.3.rs-244285/v1

License: (1) This work is licensed under a Creative Commons Attribution 4.0 International License. Read Full License

Version of Record: A version of this preprint was published at Nature Ecology \& Evolution on December 23rd, 2021. See the published version at https://doi.org/10.1038/s41559-021-01586-x. 
Spatial patterns of tumour growth impact clonal diversification: computational modelling and

3 evidence in the TRACERx Renal study

Authors:

Xiao $\mathrm{Fu}^{1 *}$, Yue Zhao ${ }^{2,3,4,5^{*}}$, Jose I. Lopez ${ }^{6}$, Andrew Rowan ${ }^{2}$, Lewis $\mathrm{Au}^{7,16}$, Annika Fendler ${ }^{7}$, Steve Hazell ${ }^{8}$, Hang Xu ${ }^{2}$, Stuart Horswell ${ }^{9}$, Scott Shepherd ${ }^{7,16}$, Lavinia Spain ${ }^{7}$, Fiona Byrne ${ }^{7}$, Gordon Stamp ${ }^{10}$, Tim O'Brien ${ }^{11}$, David Nicol ${ }^{12}$, Marcellus Augustine ${ }^{2}$, Ashish Chandra ${ }^{13}$, Sarah Rudman ${ }^{14}$, Antonia Toncheva ${ }^{15}$, Andrew Furness ${ }^{7,16}$, Lisa Pickering ${ }^{16}$, Matthew Orton ${ }^{17}$, Simon Doran ${ }^{17}$, Dow-Mu Koh ${ }^{17}$, Christina Messiou ${ }^{17}$, Derfel ap Dafydd ${ }^{17}$, Santosh Kumar ${ }^{17}$, James Larkin ${ }^{16}$, Charles Swanton ${ }^{2,3,18}$, Erik Sahai ${ }^{19 \#}$, Kevin Litchfield ${ }^{2,3 \#}$, Samra Turajlic ${ }^{7,16 \#}$ on behalf of the TRACERx Renal Consortium, Paul A Bates ${ }^{1 \#}$

1. Biomolecular Modelling Laboratory, The Francis Crick Institute, 1 Midland Rd, London NW1 1AT, UK

2. Cancer Evolution and Genome Instability Laboratory, The Francis Crick Institute, 1 Midland Rd, London NW1 1AT, UK

3. Cancer Research UK Lung Cancer Centre of Excellence, University College London Cancer Institute, Paul O'Gorman Building, 72 Huntley Street, London, WC1E 6BT, UK

4. Department of Thoracic Surgery, Fudan University Shanghai Cancer Center, Shanghai, China. 200032.

5. Department of Oncology, Shanghai Medical College, Fudan University, Shanghai, China. 200032.

6. Department of Pathology, Cruces University Hospital, Biocruces-Bizkaia Institute, 48903 Barakaldo, Bizkaia, Spain

7. Cancer Dynamics Laboratory, The Francis Crick Institute, 1 Midland Rd, London NW1 1AT, UK

8. Department of Pathology, the Royal Marsden NHS Foundation Trust, London SW3 6JJ, UK

9. Department of Bioinformatics and Biostatistics, The Francis Crick Institute, 1 Midland Rd, London NW1 1AT, UK

10. Experimental Histopathology Laboratory, The Francis Crick Institute, London NW1 1AT, UK

11. Urology Centre, Guy's and St. Thomas' NHS Foundation Trust, London SE1 9RT, UK

12. Department of Urology, the Royal Marsden NHS Foundation Trust, London SW3 6JJ, UK

13. Department of Pathology, Guy's and St. Thomas NHS Foundation Trust, London SE1 9RT, UK

14. Department of Medical Oncology, Guy's and St. Thomas' NHS Foundation Trust, London SE1 9RT, UK

38 15. Biobank, Guy's and St. Thomas' NHS Foundation Trust, London SE1 7EH, UK

39 16. Renal and Skin Units, The Royal Marsden Hospital, London, SW3 6JJ, UK

40 17. Division of Radiotherapy and Imaging, Institute of Cancer Research, 15 Cotswold 41 Road, Sutton, Surrey, SM2 5NG

42 18. Department of Medical Oncology, University College London Hospitals, 235 Euston Rd, 43 Fitzrovia, London, NW1 2BU, UK

44 19. Tumour Cell Biology Laboratory, The Francis Crick Institute, 1 Midland Rd, London NW1 45 1AT, UK 
1 Key words:

2 Computational modelling; Cellular Automaton; Clear cell renal cell carcinoma; Spatial growth 3 patterns; Microdiversity; Parallel evolution; Time-course analysis 4

5 


\section{Abstract:}

2
Intra-tumour genetic heterogeneity (ITH) fuels cancer evolution. The role of clonal diversity and genetic complexity in the progression of clear-cell renal cell carcinomas (ccRCCs) has been characterised, but the ability to predict clinically relevant evolutionary trajectories remains limited. Here, towards enhancing this ability, we investigated spatial features of clonal diversification through a combined computational modelling and experimental analysis in the TRACERx Renal study. We observe through modelling that spatial patterns of tumour growth impact the extent and trajectory of subclonal diversification. Moreover, subpopulations with high clonal diversity, and parallel evolution events, are frequently observed near the tumour margin. In-silico time-course studies further showed that budding structures on the tumour surface could indicate future steps of subclonal evolution. Such structures were evident radiologically in 15 early-stage ccRCCs, raising the possibility that spatially resolved sampling of these regions, when combined with sequencing, may enable identification of evolutionary potential in early-stage tumours. 
The development of cancer can be viewed as an evolutionary process (Merlo et al. 2006, Zahir et al. 2020). Acquisition of genomic alterations including mutations and somatic copy-number alterations (SCNAs) drives the emergence of genetically heterogeneous subpopulations of cancer cells or subclones (McGranahan and Swanton 2017), resulting in intra-tumour heterogeneity (ITH). A small subset of genomic alterations (drivers) endows subclones with increased fitness that manifests as growth and survival. Subclones compete for resources, including physical space, and undergo expansion or extinction according to their fitness under given selective pressures imposed by the tumour microenvironment (TME) or therapeutic intervention. With recent advances in next-generation sequencing, clonal architecture and evolutionary features have been elucidated in a variety of tumour types (Gerlinger et al 2012, Yates et al 2015, Jamal-Hanjani et al 2017, Turajlic et al 2018). However, the ability to predict clinically relevant evolutionary trajectories remains limited.

One potential to enhance this ability lies in the detection and characterisation of ongoing clonal evolution. ITH provides a substrate for the selection of competent clones under evolving TME and frequently underpins therapeutic failure (McGranahan and Swanton, 2015). Detection of ITH relies on proper selection of samples and can be enhanced by sampling many small-size regions (Lopez and Cortes, 2016). While ITH index and macrodiversity (i.e., the number of subclones in the whole tumour) reflect the overall degree of established clonal diversity and genetic complexity within a tumour, clone diversity at a narrow spatial scale, or microdiversity (i.e., the number of subclones within a single tumour sample), could represent under-detected ongoing clonal evolution and delineate future evolutionary trajectories (Figure 1a). Just like macrodiversity, microdiversity appears to have clinical implications and has been shown to underpin poor survival in paediatric kidney cancer (Mengelbier et al 2015). Microdiversity was also revealed by the presence of multiple subclones both in situ and in co-migrating cell groups during tumour invasion in breast tumours, using topographic single cell sequencing (Casasent et al. 2018). Clonal diversification could be facilitated by parallel evolution, that is selection of distinct mutations in the same gene in spatially separate subclones. Parallel evolution has been observed in the natural evolution of various tumour types (Gerlinger et al 2012, Turajlic et al 2018, Melchor et al. 2014, Murugaesu et al. 2015, Yates et al 2015) and in the context of therapeutic resistance under selective pressure of therapy (Voss et al 2014, Juric et al 2015). More recently, parallel evolution of SCNAs was characterised comprehensively through mirrored subclonal allelic imbalance (Watkins et al. 2020). As microdiversity and parallel evolution are important indicators of ongoing and clinically relevant tumour evolutionary features, their spatial resolution could offer further understanding of evolutionary potential.

To understand the emergence and spatial patterning of these features, we developed a coarsegrained cellular automata model of tumour growth and clonal dynamics (Figure 1b). Previous experimental evidence supports predominating proliferation at the surface of ccRCCs (Hoefflin et al. 2016). However, both some cases in this study and our recent work suggest that this may not be universally true (Zhao et al. 2020). As exemplified by several categories of mathematical models formulated to characterise tumour growth dynamics (Rodriguez-Brenes et al. 2013, Gerlee 2013), different growth patterns could have an impact on evolutionary features. Moreover, previous computational studies found an impact of growth patterns on the classification of neutral evolution and selection (Sun et al. 2017, Chkhaidze et al. 2019). Therefore, we further evaluated the effects of different spatial patterns of tumour growth (hereafter also referred to as tumour growth mode) on spatial features of clonal diversity in our 49 computational model. While distinct growth patterns may predominate in different parts of a 
1 tumour, or at different stages of the same tumour growth, we restricted our investigation in the 2 current study to two simple growth models: one model with uniform growth throughout the 3 tumour volume as a "null model" assuming that all tumour mass can proliferate (referred to as 4 "Volume Growth Model") and the other model with active proliferation restricted to the tumour 5 surface (referred to as "Surface Growth Model") (see Methods, Figure 1c).

6

In the context of the TRACERx Renal study, we reported a workflow to evaluate both the genomic profiles and spatial coordinates of 756 regions (patient tumour regions are referred to as "PT regions" hereafter) from 66 tumours (Zhao et al. 2020). Here, we focus on spatial 10 analyses of microdiversity and parallel evolution in the same dataset. We found in the model 11 setting that the spatial patterns of tumour growth influenced the extent and trajectory of subclonal diversification. Furthermore, subpopulations with high microdiversity, as well as parallel mutational events with limited clonal expansion, were frequently detected near the tumour edge, which was corroborated in the TRACERx Renal dataset. Finally, time-course studies on simulated tumours suggested that evolutionary steps could be predicted at an early stage and seeded by clones in the budding structures on the tumour surface in a subset of tumours. These budding structures were evident in 15 early-stage ccRCC tumours, raising the intriguing possibility that spatially resolved sampling, when combined with sequencing, may enable identification of evolutionary potential in early-stage tumours and predict the disease course. 
3

4

5

6

7

8

9

We developed a coarse-grained cellular automaton model to explore the evolutionary dynamics of ccRCCs, a brief outline for which is presented here (see Methods for detailed description). The model includes 12 driver genes and 14 SCNAs (Supplemental Figure 1) that were highlighted as canonical driver events in ccRCCs in the TRACERx Renal study (Turajlic et al. 2018). Each model unit, referred to as a "tumour voxel", represents a tumour volume of $1 \mathrm{~mm}^{3}$. Tumour voxels stochastically undergo growth, death, and, upon growth, processes of driver acquisition (i.e., mutations in driver genes or driver SCNAs). To keep the model minimal, several assumptions were made. In the model, time has arbitrary units, so the baseline growth probability $p_{\text {growth }}^{(1)}$ was arbitrarily defined. Three levels of growth probability, $p_{\text {growth }}^{(1)} \leq$ $p_{\text {growth }}^{(2)} \leq p_{\text {growth }}^{(3)}$, were introduced for the 26 drivers, to broadly reflect their association with the Ki67 score in tumour regions in the experimental data (Supplemental Figure 1). As one specific implementation, growth probabilities were set at $p_{\text {growth }}^{(1)}=0.25, p_{\text {growth }}^{(2)}=(1+$ $s) p_{\text {growth }}^{(1)}$ and $p_{\text {growth }}^{(3)}=(1+s)^{2} p_{\text {growth }}^{(1)}$, where $0 \leq s \leq 1$ determines this advantage relative to the baseline growth probability. For simplicity, mutations in all driver genes were assigned with the baseline growth probability, while some SCNAs were assumed to confer greater growth probabilities. Four SCNAs with strong association with Ki67 score, 7q gain, 20q gain, 4q loss, and $8 p$ loss, are the most "advantageous" drivers with $p_{\text {growth }}^{(3)}$. A tumour voxel grows with $p_{\text {growth }}^{(1)}$ conferred by the most advantageous driver harboured. Note that an alternative implementation for fitness advantage was also explored (Supplemental Figure 2, Supplemental Note 1, see Methods). Mutations in driver genes were assumed to be acquired with a greater probability $\left(p_{\text {driver }}\right)$ than SCNAs. Based on functional evidence linking them to chromosome instability (Varela et al. 2011, Peng et al. 2015), as well as their association with a high weighted genome instability index (wGII) in the experimental data from the TRACERx Renal study (Turajlic et al. 2018), mutations in PBRM1 or BAP1, were assumed to enhance the probability of SCNA acquisition. Finally, a second mutation in the same gene was assumed to never occur in the same tumour voxel.

Each simulation starts from a single tumour voxel carrying $V H L$ and $3 \mathrm{p}$ loss and is terminated when the size of the simulated tumour exceeds 1 million tumour voxels. Simulated tumours were analysed at three spatial scales: three-dimensional (3D) whole tumour volume, twodimensional (2D) tumour slice and tumour regions (Figure 1d). Exploratory simulations (50 per condition) were performed at 6 levels of $s$ and 11 levels of $p_{\text {driver }}$. This permits the understanding of the collective impact of fitness advantage and the rate of driver acquisition in a spatial context.

\section{Characterisation of clonal diversity in the whole tumour}

Tumours under Volume Growth commonly developed a single dominant subclone that grew to occupy a large proportion of tumour surface (panel (i) in Figure 1e). In comparison, tumours under Surface Growth developed multiple advantageous subclones in different regions of the tumour surface, and each of these subclones was characterised by a range of different driver events (panel (ii) in Figure 1e). Moreover, while the tumour surface in the Volume Growth 
model was smooth overall, the tumour under Surface Growth formed bulging structures on the surface, which reflected the outgrowth of driver subclones.

Given the disparity in the quantity and spatial distribution of driver subclones in the representative cases under Volume Growth and Surface Growth (Figure 1e), we then asked how the mode of tumour growth might influence the number of subclones in the whole tumour. We first counted the number of clones (including parental clone and subclones) in the whole tumour (Figure 2a-b). In the Volume Growth model, subclones were only observed in tumours with greater $s$ and greater $p_{\text {driver }}$ (panel (i) in Figure 2b). By contrast, in the Surface Growth model, tumours with small to moderate $p_{\text {driver }}$ harboured more subclones, for a wide range of $s$ (panel (ii) in Figure 2b). These findings suggest that the mode of tumour growth could impact outgrowth of subclones and their ultimate prevalence.

Noticeably, tumours also displayed distinct proportions of subclonal populations, independent of the growth mode and evolutionary conditions (i.e., identical $s$ and $p_{\text {driver }}$ ) (Figure $2 \mathbf{c}$ ). In the Volume Growth model, most tumours with a small $p_{\text {driver }}$ displayed a monoclonal structure with limited evidence of clonal evolution (panel (i) in Figure 2c). With a large $p_{\text {driver }}$, a greater extent of clonal evolution was evident in a higher proportion of tumours (panel (ii) in Figure 2c). In a fraction of these cases, one dominant subclone was observed with the parental clone present as a minority, suggesting early fixation of a highly fit clone and a (near) clonal sweep (i.e., the entire tumour was taken up by the dominant subclone). In comparison, in the Surface Growth model, extensive subclonal diversification was evident in nearly all cases, even with a small $p_{\text {driver }}$ (panel (iii) in Figure 2d). These features observed in individual tumours were reflected by the whole-tumour cancer cell fraction (CCF) of the parental clone (Figure 2e) and Shannon diversity index (Figure 2f). Across a wide range of driver acquisition probabilities, the Surface Growth model, in comparison to the Volume Growth model, consistently displayed a relatively lower whole-tumour CCF of the parental clone and a greater Shannon diversity index, indicating a greater extent of subclonal diversification. The greater extent of diversification in the Surface Growth model was also noted for conditions with still smaller $p_{\text {driver }}$ (Supplemental Figure 3) or smaller $s$ (Supplemental Figure 4), where clonal evolution was generally limited. For the interest of characterising patterns of subclonal diversification and contrasting two growth modes, we limited our parameter analysis, for the subsequent investigation, to $s=1$ and a range of $p_{\text {driver }}$ from $2 \times 10^{-4}$ to $1 \times 10^{-3}$.

In general, Surface Growth appears to enable more extensive subclonal diversification, leading to a highly branched tumour evolution, while Volume Growth often resulted in tumours with limited clonal evolution and occurrence of punctuated evolution. Interestingly, these were the precise modes of evolution that underlined diverse evolutionary subtypes identified in ccRCCs, ranging from "VHL mono-driver" tumours with limited evidence of clonal evolution, to tumours with extensive subclonal diversification characterised by a range of drivers, and those with early fixation of a highly fit clone resulting in rapid clonal sweep (Turajlic et al. 2018).

Having established a quantitative understanding of clonal diversity in the whole tumour and the impact of different growth modes, we next strived for a spatial understanding of clonal diversity. 
As a first step towards understanding spatial features of clonal diversity, we asked whether insilico tumours were able to recapitulate a phenomenon of spatial clonal evolution noted in colorectal and liver cancers by others (Cross, et al. 2017; Zhai, et al. 2017) and, more recently, in ccRCCs by our group (Zhao et al. 2020), whereby subclones are patterned within a tumour such that regions farther apart exhibit more divergent genomic makeups.

To this end, we sampled uniformly spaced regions within a 2D tumour slice and measured the spatial and genomic distances between each pair of regions (Figure 3a, see Methods). Consistent with previous experimental evidence (Cross, et al. 2017; Zhai, et al. 2017; Zhao, et al. 2020), between-region spatial and genomic distances were positively correlated (hereafter referred to as "spatial-genomic correlation") in representative tumours under Surface Growth (Figure 3b-c) or under Volume Growth (Figure 3d-e) and also in repeat simulations across multiple values of $p_{\text {driver }}$ (Figure 3f). Moreover, different growth modes resulted in distinct trends of variation in the spatial-genomic correlation with the change of $p_{\text {driver }}$. In the Volume Growth model, the spatial-genomic correlation was weaker in tumours with smaller $p_{\text {driver }}$. By contrast, in the Surface Growth model, the spatial-genomic correlation was weaker in tumours with greater $p_{\text {driver }}$, as the extensive diversification likely caused large genomic divergence even in regions separated by a short distance (Figure 3f). As a consequence of these divergent trends, the spatial-genomic correlation was stronger in tumours under Surface Growth than those under Volume Growth if $p_{\text {driver }}$ was small, and weaker if $p_{\text {driver }}$ was large. Experimentally, the cohort of ccRCCs in the TRACERx Renal study displayed a spatialgenomic correlation coefficient of 0.31 (Zhao, et al. 2020).

Overall, these data demonstrate that our model is sufficient to recapitulate the spatial-genomic correlations observed across experimental data, a pattern emergent from spatial evolution of subclones in tumours. We next asked how clonal diversity itself could vary spatially within a tumour slice and whether the mode of tumour growth had an impact on the spatial patterns.

\section{Spatial distribution of clonal diversity within tumour sections}

Characterisation of clonal diversity in the whole tumour and the between-region genomic divergence provided a quantitative view of the extent of subclonal diversification. We next sought to examine whether clonal diversity was spatially uniform or variable within a tumour. To this end, a $3 \mathrm{~mm}$-by-3 $\mathrm{mm}$ sliding window was applied throughout the $2 \mathrm{D}$ tumour slice and the number of distinct subclones (defined as "microdiversity") was counted in that 9- $\mathrm{mm}^{2}$ area (Figure 4a). Subsequently, the spatial profile of microdiversity was analysed to identify hotspots with microdiversity equal to or greater than 5 (defined as "microdiversity hotspots") (Figure 4a).

Consistent with the previous analysis on the whole-tumour CCF of subclones (Figure 2a), tumours under Surface Growth developed multiple subclones that occupied distinct spatially contiguous areas, reflective of a branched clonal structure (panel (i) in Figure $4 \mathbf{b}$ ). Microdiversity hotspots were frequently observed near the tumour edge within outgrowing advantageous subclones but also at the boundaries of multiple adjacent subclones (panel (ii) in Figure 4b). By contrast, tumours under volume growth commonly developed a single dominant subclone (panel (i) in Figure 4c). In these tumours microdiversity hotspots were 
1 observed within the area spanned by the dominant subclone with a more uniform distribution 2 along the tumour radius (panel (ii) in Figure 4c).

3 To corroborate these spatial features of clonal diversity with experimental data, we examined 466 tumours in the TRACERx Renal study. Individual PT regions that contain at least two subclones were treated as a proxy for microdiversity hotspots. In total, 606 PT regions from 54 tumours were included in this analysis. Different spatial distributions of microdiversity hotspots were observed, as highlighted in representative examples: predominantly near the tumour margin (as in "G_K234"), while more uniformly distributed throughout the tumour section (as in "G_K446") (Figure 4d).

In order to quantify the observed spatial patterns, we measured the distance to the tumour centre and the distance to the closest point at the tumour contour, for every microdiversity hotspot over all repeat simulations. These two distances were then combined into a single variable termed the "normalised distance to tumour centre", denoted as $d$. Interestingly, the cumulative probability distribution with respect to $d$ depicted power law scaling (Figure 4e), suggesting that the probability of observing spots with high clonal diversity along the radius of a tumour could be estimated using a simple mathematical formula (i.e., $P(D \leq d) \sim d^{k}$, where $k$ is the power law exponent to be fitted). Furthermore, the Surface Growth model displayed a larger $k$ compared to the Volume Growth model across multiple values of $p_{\text {driver }}$ (Figure 4f, Supplemental Figure 5), indicating a greater likelihood of microdiversity hotspots being located near the tumour edge under Surface Growth. The power law pattern characterising the spatial distribution of microdiversity hotspots was corroborated in the experimental data (Figure 4e); ccRCC tumours displayed a $k$ value numerically between those in the Volume Growth and Surface Growth models (Figure 4g). A greater error in matching data and fitted power law was noted at regions approaching the margin, likely attributed to under-sampling of biopsies at the tumour periphery (Supplemental Figure 5).

To summarise, regions with high clonal diversity within a tumour slice were increasingly frequent towards the tumour margin, a spatial feature particularly enhanced in the Surface Growth model and corroborated experimentally using PT regions with at least two subclones. These data suggested that subpopulations with high clonal diversity were abundant near the tumour margin.

Frequency and spatial features of parallel evolution

As subclonal diversification could involve acquisition of, and be facilitated by, distinct mutations in the same gene at spatially separate locations, we next evaluated the frequency of parallel evolution events and their spatial features.

Distinct mutational instances in the same driver gene were recorded as different driver events in the model (Figure 5a). Guided by an exploratory whole-tumour analysis, which showed parallel mutational events other than PBRM1 and BAP1 to be rare at relatively low resolution (Supplemental Note 2, Supplemental Figure 6a-b), we specifically focused on these two mutations. As might be expected, as a reflection of the extent of subclonal diversification in the whole tumour shown above (Figure 2b), parallel evolution was evident in the Volume Growth model only when $s$ and $p_{\text {driver }}$ were both large (panel (i) in Figure 5b), while it was pervasive in the Surface Growth model under various parameter conditions (i.e., pairs of $s$ and $p_{\text {driver }}$ ) (panel (ii) in Figure 5b).

48 To further elucidate the spatial patterns of parallel mutations in PBRM1 or BAP1, we next 49 labelled tumour regions harbouring these events (Figure 5a). Within a tumour section, 
subpopulations harbouring parallel mutational events expanded to occupy a variable number of regions (Figure 5c-d). When mutational instances in PBRM1 or BAP1 occurred late, they led to limited clonal expansion (up to two tumour regions), despite the role in promoting acquisition of SCNAs (Supplemental Figure 6c-f). Those instances were commonly observed at a short distance to the tumour edge (Figure 5e). Furthermore, for a range of $p_{\text {driver }}$, the Surface Growth model resulted in a shorter distance of the parallel evolution events to the tumour edge, compared to the Volume Growth model, likely due to the birth of these subclones occurring at the tumour surface.

In the TRACERx Renal study, parallel evolution was observed in 28 tumours, with parallel mutations in the same gene spanning distinct sets of regions (Supplemental Note 3). As highlighted previously (Turajlic, et al. 2018) and similar to the in-silico tumours, distinct parallel mutational events could span a variable number of PT regions (Figure 5f).

Consistent with the in-silico analyses, parallel mutational events in driver genes with limited clonal expansion (spanning up to two PT regions) were predominantly located near the tumour edge (Figure 5g). This spatial distribution was also found for mutational events without evidence of parallel evolution and with limited expansion (Figure 5g), suggesting that subclones lacking significant expansion were commonly emerging near the tumour edge. The lack of expansion of these subclones could be attributed to either their narrow fitness advantage and/or late occurrence.

Together, these data suggest that parallel evolution in $B A P 1$ or $P B R M 1$ was more prevalent in the Surface Growth model, which underlined extensive subclonal diversification. For both insilico modelling and experimental analysis of ccRCC tumours, parallel mutations with limited clonal expansion were located near the tumour edge, implying that a spatially resolved sampling could target the tumour margin to identify ongoing parallel evolution.

\section{Predictive feature of future evolutionary trajectories}

Having so far focused on quantitative and spatial characterisation of microdiversity and parallel evolution, we next asked how features of clonal diversity evolved temporally and whether some features could enable the prediction of future evolutionary trajectories. The ability to predict likely evolutionary trajectories could aid in the clinical management of ccRCC tumours. However, the challenges of obtaining serial biopsies of the same tumour limit this opportunity in clinical practice. Therefore, we examined if our model could be used to indicate the temporal features of evolution of clonal diversity.

To this end, the number of subclones within a tumour slice was tracked over time. Tumours under Volume Growth developed a small number of subclones that were sustained over the course of tumour evolution (Figure 6a, panel (i) in Figure 6b, Supplemental Figure 7a). By comparison, the number of subclones increased over time in tumours under Surface Growth (Figure 6a, panel (ii) in Figure 6b, Supplemental Figure 7b). Nevertheless, tumours under distinct growth modes appeared indistinguishable at early stage according to this measurement. We then asked whether some spatial features could indicate early the divergent patterns of subsequent subclonal diversification in the two models.

To shed light on this question, we evaluated the Surface Growth model for the presence of early indicators of subclonal diversification. In fact, one characteristic spatial feature noticed in tumours under Surface Growth was a budding structure on the tumour surface, which indicated the beginning of outgrowth of an advantageous subclone (Figure 1e, Figure 6c). 
1 Quantitatively, this budding structure reflected the rapid increase in the CCF of an 2 advantageous subclone, as it spatially outcompeted surrounding subpopulations (Figure 6d). 3 Depending on the driver acquisition probabilities employed in the model, the median tumour 4 size for the detection of such budding structures was around $7 \mathrm{~cm}$ or smaller. Exploratory simulations attempting at "replaying" evolution (i.e., re-simulating clonal evolution from a historical tumour state with established clonal structure as a starting point) starting from different tumour sizes suggested that evolution was more repeatable if starting from a historical tumour state with budding structures emerging (Supplemental Figure 8, Supplemental Note 4).

With respect to the above findings, we then turned to analysing 46 tumours with a size of up to $7 \mathrm{~cm}$ in the TRACERx Renal study. By qualitative examination of radiological images of these tumours, 15 tumours displayed apparent budding structures at their surface. In one representative case ("G_K523"), budding structures were evident both radiologically (Figure 6e) and in the tumour contour image with clonal diversity mapped (Figure 6f). Interestingly, trailing the budding structures were regions with high clonal diversity, consistent with our insilico tumour simulations under Surface Growth. These findings imply ongoing subclonal diversification in these regions that may delineate future evolutionary steps.

Lastly, in the TRACERx Renal study, the number of subclones depicted a non-linear relationship with tumour size (Figure 6g). This broadly reflected different modes of evolution, ranging from limited evolution (bottom-left part in Figure $\mathbf{6 g}$ ), to punctuated evolution (bottom-right part in Figure 6g) and branched evolution (top part in Figure 6g). Furthermore, a subset of tumours (enclosed only by blue contour in Figure 6g) were better recapitulated by the Volume Growth model, while another subset by Surface Growth model (enclosed only by red contour in Figure 6g). A more intriguing question still is whether the future steps of clonal evolution in the early-stage tumours could be predicted. When 15 early-stage tumours with apparent budding structures were highlighted, a subset of these already displayed a greater extent of subclonal diversification, raising the possibility that spatially resolved sampling combined with sequencing in these regions may enable identification of evolutionary potential in early-stage tumours. 
Intra-tumour genetic heterogeneity arises when different parts of the tumour acquire distinct genomic alterations, endowing subclones with a variety of fitness advantages. Despite our understanding of how dominant subclones sculpt evolutionary trajectories and histories, gleaned primarily from multi-region sampling and deep sequencing, under-represented subclones that reflect ongoing and clinically relevant evolution could remain undetected. Therefore, our focus on microdiversity and parallel evolution is central to the elucidation of the evolutionary potential of under-represented subclones and the emergent spatial patterns they form.

An important finding, via computational modelling, is that different spatial patterns of tumour growth impact the extent of subclonal diversification and shape divergent modes of evolution. While Surface Growth (i.e., proliferation limited to the superficial layer of a tumour) enables more extensive subclonal diversification and gives rise to branched evolution, Volume Growth (i.e., proliferation active throughout the tumour volume) commonly results in single dominant subclone coexistent with founding clone reflective of punctuated evolution. Linear evolution was not observed in our model. Experimentally, the inferences of linear evolution are generally biased by the lack of sufficient sampling to detect intermediate populations. Overall, this observation resonates with other computational studies to highlight the importance of understanding spatial patterns of tumour growth when deconstructing tumour evolution (Anderson et al 2006, Waclaw et al 2015, Sun et al 2017, Chkhaidze et al 2019, Noble et al 2019).

Another important finding, in both the in-silico tumours, in particular those under Surface Growth, and tumour in the TRACERx Renal study, are frequent microdiversity hotspots and parallel mutational events near the tumour edge, suggesting abundant ongoing evolution at the tumour margin. Temporally tracking the parallel mutational events in PBRM1 or BAPl in the in-silico setting illuminated the rapid increase of their prevalence in small tumours leading to ultimately established subclones. In those under Surface Growth, this rapid transition was marked by a budding structure on the tumour surface. Interestingly, such budding structures were evident in 15 early-stage ccRCC tumours. While a prospective study in a large cohort is needed for further examination, this observation opens up the possibility that designing spatially resolved sampling strategies in early-stage tumours with assessment of clonal diversity at the tumour margin, in particular within the budding structures, could be beneficial in informing upon a tumour's evolutionary potential.

Our computational modelling also uncovered power law scaling features that uniquely characterise clonal diversity in a spatial context. These features add to previously reported scaling phenomena describing clonal evolution (Driessens et al 2012, Williams et al 2016). In addition to the scaling feature underlying the spatial distribution of microdiversity hotspots, clonal diversity and sampling area also form a scaling relationship in both the model and experimental data (Supplemental Figure 9, Supplemental Note 5). This scaling relationship in ccRCC tumours is reminiscent of the taxa-area relationship in the macroevolution of species (Crawley and Harral 2001, Horner-Devine et al 2004, Zhou et al 2008), drawing a parallel between cancer evolution and evolution in the ecosystem.

Overall, our computational model was able to correlate with several clinical observations, despite the simplification of its primary components (probabilistic birth, death, and driver acquisition). Nevertheless, we are aware that our model has limitations. Clearly, many other 
1 factors, such as vascularisation, necrosis, and immune predation, are all likely to (re)shape the 2 in-silico patterns of clonal diversity we observed. The simple implementations of fitness 3 advantage endowed by ccRCC drivers could be improved in the future with wet-lab 4 experiments aimed at measuring and comparing growth kinetics of tumours with different 5 genetic backgrounds. Future time-lapse experiments could also provide evidence for growth 6 modes of ccRCCs and elucidate whether different modes may predominate in different regions 7 or at different stages of the same tumour. Moreover, while we made an assumption that only 8 mutations in $B A P 1$ or $P B R M 1$ promoted acquisition of SCNAs for simplicity, we acknowledge 9 that other ccRCC driver mutations (e.g., SETD2) also link to chromosome instability. Lastly, 10 alternative mechanisms could explain the formation of budding structures and other protrusive 11 morphologies, such as mechanical properties (Fiore et al. 2020) and cell migration (Anderson 12 et al. 2006). In addition to these limitations from a modelling perspective, differences in size, 13 stage, and the number of samples among tumours in the TRACERx Renal study could 14 confound the patterns to which the computational outputs are compared. The current 15 sequencing data is panel-based with a small number of clonal markers and may have low 16 sensitivity to detect microdiversity. Future examination of whole-exome or whole-genome data

19 In conclusion, our study supports the importance of understanding spatial patterns of tumour 20 growth in deconstructing tumour evolution. It provides evidence, and has implications for, 21 focused spatial sampling of the tumour margin in order to garner richer information on ongoing 22 and clinically relevant evolution. 
3 Tumour growth and clonal evolution in a spatio-temporal context have increasingly been 4 studied with the aid of computational models that incorporate rule-based spatial growth and

\section{Growth and death} Chkhaidze et al 2019). Spatial patterns of tumour growth (Sun et al 2017, Chkhaidze et al 2019) have been shown to impact the ability to classify neutral evolution in contrast to selection, suggesting that spatial growth of a structured population interplays with evolutionary forces (driver acquisition, selection, and genetic drift) to shape the spatial patterning of subclones.

In the present study, a coarse-grained cellular automaton model has been constructed to simulate tumour growth and the evolution of ccRCC drivers. A basic model unit reflects a tumour volume of $1 \times 1 \times 1 \mathrm{~mm}^{3}$, referred to as a "tumour voxel". The choice of $1 \mathrm{~mm}$ for the size of a tumour voxel reflects the observed thickness of actively proliferative surface layer of some ccRCCs (Hoefflin et al. 2016). The full simulation lattice comprises $200 \times 200 \times 200$ lattice sites in which a tumour grows. The following sub-sections detail the model components and assumptions.

Tumour voxels stochastically undergo growth and death, with baseline probabilities per simulation step of $p_{\text {growth }}=0.25$ and $p_{\text {death }}=0.05$, respectively. Upon death, a tumour voxel is removed from the simulation lattice, rendering the site empty and available for accommodating new tumour voxels. Two different modes of spatial tumour growth are considered: Surface Growth and Volume Growth (Figure 1c). For Surface Growth, proliferation is only allowed to take place when space is available, namely, when at least one of the 26 neighbouring lattice sites of the tumour voxel selected to divide is empty. Upon duplication of a parent tumour voxel, one child tumour voxel retains the location of the parent while the other is placed at a randomly selected adjacent empty site. For Volume Growth, all tumour voxels are able to proliferate; upon duplication, one child tumour voxel retains the location of the parent while the other is placed at a selected adjacent site according to the rule described below and pushes tumour voxels in that orientation outward. The process for selecting an adjacent site includes two steps: (1) to randomly sample 10 candidate positions out of the 26 neighbouring lattice sites; (2) to select the orientation (i.e., pointing from the position of the parent tumour voxel to the candidate position) giving the smallest distance from the tumour surface, similar to the algorithm described in Waclaw et al. 2015.

\section{Driver events}

A panel of 26 ccRCC drivers that were highlighted in Turajlic et al. 2018, including mutations in 12 driver genes and 14 somatic copy number alterations (SCNAs), are considered in the present work (Supplemental Figure 1). For simplicity, the fitness advantage conferred by a driver is assumed to manifest as growth advantage. In implementation, three levels of growth probability, $p_{\text {growth }}^{(1)} \leq p_{\text {growth }}^{(2)} \leq p_{\text {growth }}^{(3)}$, were introduced for the 26 drivers, to broadly reflect their association with the Ki67 score in tumour regions in the experimental data 
(Supplemental Figure 1). In a general form, $p_{\text {growth }}^{(2)}=g(s) p_{\text {growth }}^{(1)}$ and $p_{\text {growth }}^{(3)}=$ $2 h(s) p_{\text {growth }}^{(1)}$ are functions of baseline growth probability, where $h(s) \geq g(s) \geq 1$ reflect the 3 growth advantages relative to the baseline. As one specific implementation, growth 4 probabilities were set at $p_{\text {growth }}^{(1)}=0.25, p_{\text {growth }}^{(2)}=(1+s) p_{\text {growth }}^{(1)}$ and $p_{\text {growth }}^{(3)}=(1+$ $s)^{2} p_{\text {growth }}^{(1)}$, where $0 \leq s \leq 1$ determines this advantage relative to the baseline growth 6 probability. The baseline growth probability $p_{\text {growth }}^{(1)}$ was arbitrarily defined, as time has 7 arbitrary units in the model. For a given tumour voxel, its growth probability is defined by the 8 most advantageous driver harboured (i.e., "saturated" model of fitness advantage). For 9 simplicity, VHL mutation and $3 p$ loss are considered as truncal events in the founder tumour voxel, although in a clinical setting $V H L$ mutation are not present universally (Turajlic et al. 2018). The subpopulation of tumour voxels that only harbour these two events is referred to parental clone. For an exploratory purpose, an alternative implementation of growth advantage endowed by drivers was also evaluated (Supplemental Figure 2). In this implementation, a driver adds a certain amount of growth probability to the tumour voxel that acquires the driver (i.e., "additive" model of fitness advantage) (Supplemental Figure 2a). The growth probability of a tumour voxel depends on all the drivers it harbours, namely, $p_{\text {growth }}=$ $p_{\text {growth }}^{(1)}+\sum_{k} p_{\text {growth_k }}$, where $p_{\text {growth_k }}$ reflects the amount of growth probability added by

Upon proliferation of a parent tumour voxel, child tumour voxels inherit existing driver events harboured by the parent tumour voxel and stochastically acquire new drivers. Mutations in driver genes are acquired with a probability $p_{\text {driver }}$ and are assumed to be acquired in a tumour voxel more frequently than SCNAs, which have probability of $0.001 p_{\text {driver }}$. Given the functional evidence that BAPl (Peng et al. 2015) and PBRMI genes (Varela et al. 2011) guard against chromosome instability and the experimental data in the TRACERx Renal study demonstrating the association of mutations in these genes with high wGII (Turajlic et al. 2018), we assume that the acquisition of SCNAs in a tumour voxel becomes equally likely as mutations in driver genes, if the tumour voxel harbours mutations in BAPl or PBRM1. A range of driver acquisition probabilities have been studied to explore its impact on patterns we investigate. Lastly, only one mutation in the same driver gene is permitted in the same tumour voxel, but multiple independent, distinct mutations may be acquired in parallel within a simulated tumour in different tumour voxels.

Each simulation starts from a single tumour voxel placed at the centre of the lattice, $\left(x_{0}, y_{0}, z_{0}\right)$, and runs until the tumour grows to at least 1 million tumour voxels after the last simulation step. In each simulation step, growth and death are evaluated for every tumour voxel, in random order. For those tumour voxels selected to grow, driver acquisition is evaluated for each of the 26 drivers. The computer code is written in CUDA $\mathrm{C}++$. 
3 Analyses have been conducted at three different levels (Figure 1D): (1) whole tumour level, 4 which takes into account all tumour voxels in the 3D volume; (2) tumour slice level, which 5 takes into account all tumour voxels within a 2D plane $\left(z=z_{0}\right)$; (3) regional biopsy level, 6 which takes into account tumour voxels within regional biopsies. A regional biopsy is defined 7 as all tumour voxels within a region in the 2D slice. Spatially uniform sampling is performed 8 in this study. This process is carried out by locating the centres of candidate regional biopsies 9 in the $200 \mathrm{~mm} \times 200 \mathrm{~mm}$ 2D lattice with a spacing of $20 \mathrm{~mm}$ and collecting all voxels within 10 a distance of $5 \mathrm{~mm}$ from each biopsy centre.

Spatial maps of driver events

In representative cases, tumour voxels that harbour selected driver events are mapped, within a 3D tumour surface and within a 2D tumour slice. In spatial maps of driver events, tumour voxels that don't harbour drivers of interest are in grey; tumour voxels that harbour mutations in driver genes are in blue; tumour voxels that harbour gain of chromosome arm events are in green; tumour voxels that harbour loss of chromosome arm events are in red.

\section{Spatial maps of subclones}

A subclone is defined as a group of tumour voxels that harbour the same set of driver events. In spatial maps of subclones, tumour voxels that belong to the parental clone are in grey, while other subclones are visualised in randomly generated (R, G, B) colours.

\section{Cancer cell fraction (CCF) of driver events}

$\mathrm{CCF}$ of a driver event is calculated as the number of tumour voxels that contain the driver event divided by the total number of tumour voxels in the domain of interest, depending on the level of analyses. A driver event is considered detectable if the CCF is greater than 0.01 .

\section{Cancer cell fraction of subclones}

CCF of a subclone is calculated as the number of tumour voxels that belong to a subclone divided by the total number tumour voxels in the domain of interest, depending on the level of analyses. A subclone is identified by a set of driver events, shared by a subpopulation of tumour voxels, which are accumulated within the subclone-initiating tumour voxel. A subcloneinitiating tumour voxel is defined as a tumour voxel that acquires a new driver event upon birth. A subclone is considered detectable if the CCF is greater than 0.01 .

\section{Shannon diversity index}

38 As a measure of clone diversity, Shannon diversity index is defined as $S=\sum_{i}-f_{i} \ln f_{i}$, where $39 f_{i}$ is the CCF of the subclone $i$. All subclones are taken into account in this calculation. 
2 Spatial distance between two regional biopsies is calculated as the Euclidean distance between 3 the positions of the centres of two biopsies. Genomic distance between two regional biopsies

4 is calculated as $d_{g e n}=\sqrt{\sum_{k}^{26}\left(G_{i}^{k}-G_{j}^{k}\right)^{2}}$, where $G_{i}^{k}$ is the state of $k$ th driver in the $i$ th region

\section{Microdiversity}

Microdiversity is defined as the number of subclones contained in a 3-mm-by-3-mm region within the tumour slice. In representative cases, microdiversity is spatially mapped within a tumour slice, by sliding a 3-mm-by-3-mm spatial window throughout the tumour slice. Microdiversity hotpots are defined as a subset of these small regions with 5 or more subclones. The distance from a microdiversity hotspot to the centre of a tumour slice is referred to as the distance to tumour centre $\left(d_{1}\right)$. The distance from a microdiversity hotspot to the nearest point along the tumour contour is referred to as the distance to tumour margin $\left(d_{2}\right)$. The normalised distance to tumour centre is defined as $d=d_{1} /\left(d_{1}+d_{2}\right)$. Cumulative probability distribution of $d$ is generated by combining microdiversity hotspots from repeat simulations. Power law exponent is obtained by bootstrapping 100 samples of 400 hotspots per sample and fitting a power law function to cumulative probability distribution of $r$ in each sample.

Parallel evolution refers to the independent, distinct instances of mutations in the same driver gene that are acquired at different tumour locations. At the whole tumour level, the extent of parallel evolution is assessed by counting the instances of independent, distinct instances of mutations in each driver gene at a given resolution of detection. The resolution of detection is represented using a minimum CCF that has to be reached by a parallel mutational event to be detectable. At the regional biopsy level, the extent of parallel evolution is assessed by counting the number of regions that contain distinct instances of mutations for each driver gene. The distance from any region containing a parallel instance to the tumour margin is measured, to study the spatial distribution of parallel mutational instances with limited clonal expansion.

Temporal analysis

For the time-course study, 250 simulations with 5 different levels of $p_{\text {driver }}$ (50 simulations per condition) are performed for each of Surface Growth and Volume Growth models. 2D tumour slices are collected every 10 steps in Surface Growth model and 5 steps in Volume Growth model. The number of subclones is counted within each historical tumour slice. Kernel density estimation with a Gaussian kernel is performed with respect to the number of subclones and the diameter of the tumour slice, based on all simulations, to produce a continuous density estimate, using seaborn. jointplot ( $\mathrm{kind}=$ ' $\mathrm{kde}$ ') in Python. In a representative case, tumour voxels that harbour parallel mutational events in BAPl are mapped within historical tumour slices. In all simulations, CCFs of subclones that harbouring mutations in BAP1 are measured within historical tumour slices over time. 
2 To perform evolutionary replay, historical tumour state (i.e., locations and clonal identities of 3 tumour voxels) at a certain time point is saved and employed as a common starting state for re4 growth 50 new simulated tumours. At the end of these simulations, Shannon diversity index is 5 calculated to indicate the divergence in their evolutionary outcomes.

Experimental analyses

8

9

\section{TRACERx Renal cohort}

79 tumour sections of 66 unique primary tumours are included in this study; see the exclusion criterion in our previous publication (Zhao, et al. 2020).

\section{CT images}

Contrast-enhanced CT images were obtained using standard-of-care imaging sequences in 91 patients and curated using a local research PACS (based on the XNAT platform, (Marcus, et al., 2007)). Outlines were drawn giving volumetric tumour coverage by an oncologist (S.S.) and checked by a radiologist (D.A.), from which image strips were prepared for rapid visualisation of all tumour slices for all patients using an in-house script written in python.

\section{Microdiversity}

Spatial maps of regional clone diversity are created for two representative tumour sections. In these maps, regions are colour-coded based on the number of subclones. Regions that harbour at least one subclone are treated as a proxy for microdiversity hotspots defined in the model analysis. In total, there are 606 regions from 54 tumours that satisfy this criterion. For these regions, the normalised distance to tumour centre is measured as described above in "Model analysis - Microdiversity".

\section{Parallel evolution}

Spatial maps of parallel mutational events in PBRM1 are created for one representative tumour section. In these maps, regions are coloured differently according to different parallel mutational events. Regions that harbour more than one event are indicated with multiple colours. To study the spatial distribution of mutational events with limited clonal expansion, the maximum distance from an event spanning up to two regions to the tumour margin is measured.

\section{Statistical analysis}

Two-sided Wilcox's rank test is performed to compare several measurements between different model conditions. Statistical significance is annotated within box plots using stat_compare_means (method = "wilcox.test", label = "p.signif") in $\mathrm{R}$. 
1 Pearson correlation coefficients are calculated to assess the correlation between spatial and 2 genomic distances between tumour regions, using cor.test(variable1, 3 variable2, method = "pearson") in R.

4 Two-sample Kolmogrov-Smirnov test is performed to compare two cumulative probability 5 distributions, using scipy.stats.ks 2 samp (sample1, sample2) in Python.

6 Bootstrapping is performed to generate 100 random samples of 400 microdiversity hotspots

7 per sample with replacement, using random. choice () in Python. The power law

8 exponent is then determined by fitting a power law function to the cumulative probability

9 distribution from each sample, using scipy.optimize.curve_fit() in Python.

10 Quantile-Quantile (Q-Q) plot is employed to compare actual distribution of microdiversity

11 hotspots with a power law distribution with exponent being the median of fitted values in

12 bootstrapping, using statsmodels.graphics.gofplots.qqplot () in Python.

13 Kernel density estimation is performed for simulations with respect to the size of tumour

14 slice and the number of subclones, using seaborn.jointplot (kind="kde") in

15 Python.

$16 \quad \mathrm{R}$ version 3.6.2 and Python version 3.7.7 are used for these analyses.

17

18 Code availability

19 CUDA $\mathrm{C}++$ code of a representative model is available on GitHub: https://github.com/xxxxx 20 
This research was funded in whole, or in part, by the Wellcome Trust (FC001003). For the purpose of Open Access, the author has applied a CC BY public copyright licence to any Author Accepted Manuscript version arising from this submission.

This work was supported by the Francis Crick Institute, which receives its core funding from Cancer Research UK (FC001003, FC001144, FC001169, FC001988), the UK Medical Research Council (FC001003, FC001144, FC001169, FC001988), and the Wellcome Trust (FC001003, FC001144, FC001169, FC001988).

We thank colleagues from Biomolecular Modelling Lab, Tumour Cell Biology Lab, Cancer Dynamics Lab, and Cancer Evolution and Genome Instability Lab for insightful discussion and comments on this manuscript.

ST is funded by Cancer Research UK (grant reference number C50947/A18176); the Francis Crick Institute, which receives its core funding from Cancer Research UK (FC10988), the UK Medical Research Council (FC10988), and the Wellcome Trust (FC10988); the National Institute for Health Research (NIHR) Biomedical Research Centre at the Royal Marsden Hospital and Institute of Cancer Research (grant reference number A109); the Royal Marsden Cancer Charity; The Rosetrees Trust (grant reference number A2204), Ventana Medical Systems Inc (grant reference numbers 10467 and 10530); the National Institute of Health (Bethesda) and Melanoma Research Alliance. ST has received speaking fees from Roche, Astra Zeneca, Novartis and Ipsen. ST has the following patents filed: Indel mutations as a therapeutic target and predictive biomarker PCTGB2018/051892 and PCTGB2018/051893 and Clear Cell Renal Cell Carcinoma Biomarkers P113326GB. A.F. receives funding from the European Union's Horizon 2020 research and innovation programme under the Marie Skłodowska-Curie grant agreement No. 892360.

We thank the TRACERx Renal trial team and the Skin and Renal Unit Research Team at The Royal Marsden NHS Foundation Trust, including Eleanor Carlyle, Lyra Del Rosario, Kim Edmonds, Karla Lingard, Mary Mangwende, Sarah Sarker, Charlotte Lewis, Fiona Williams, Hamid Ahmod, Tara Foley, Dilruba Kabir, Justine Korteweg, Aida Murra, Nahid Shaikh, Kema Peat, Sarah Vaughan and Lucy Holt. TRACERx Renal is funded by NIHR BRC at the Royal Marsden Hospital and Institute of Cancer Research (A109). The Francis Crick Institute, which receives its core funding from CRUK (FC010110), the UK Medical Research Council (FC010110), the Wellcome Trust (FC010110).

\section{The TRACERx Renal Consortium}

The members of TRACERx Renal Consortium are: Lewis Au, Ben Challacombe, Ashish Chandra, Simon Chowdhury, William Drake, Archana Fernando, Nicos Fotiadis, Andrew Furness, Emine Hatipoglu, Karen Harrison-Phipps, Steve Hazell, Peter Hill, Catherine Horsfield, James Larkin, Jose I. Lopez, Teresa Marafioti, David Nicol, Tim O’Brien, Jonathon Olsburgh, Lisa Pickering, Alexander Polson, Sergio Quezada, Sarah Rudman, Scott Shepherd, Charles Swanton, Samra Turajlic, Mary Varia, Hema Verma. 
2 X.F., Y.Z., E.S., K.L., S.T., and P.A.B. conceived and designed the study.

3 X.F. and P.A.B. constructed the computational model and performed simulations.

4 Y.Z., C.S., K.L., and S.T. provided the tumour samples and performed imaging analysis.

5 S.D., M.O, and S.K curated and processed the radiological data in this study. S.S. performed

6 the initial outlining of the tumours and D.A. checked and refined them.

$7 \quad$ X.F. performed data analysis on simulations.

8 X.F., Y.Z. and J.I.L performed data analysis on tumour samples.

9 X.F., S.T., and P.A.B. wrote the manuscript with input from all authors.

11 K.L., S.T., and C.S. have a patent on indel burden and checkpoint inhibitor response pending, and a patent on targeting of frameshift neoantigens for personalised immunotherapy pending. K. L. reports speaker fees from Roche Tissue Diagnostics. ST reports grants from Ventana, outside the submitted work. J.L. reports institutional research support from: BMS, MSD, Novartis, Pfizer, Achilles Therapeutics, Roche, Nektar Therapeutics, Covance, Immunocore, Pharmacyclics, Aveo, and consultancy support from: Achilles, AZ, Boston Biomedical, BMS, Eisai, EUSA Pharma, GSK, Ipsen, Imugene, Incyte, iOnctura, Kymab, Merck Serono, MSD, Nektar, Novartis, Pierre Fabre, Pfizer, Roche / Genentech, Secarna, Vitaccess. C.S. receives grant support from Pfizer, AstraZeneca, BMS, Roche-Ventana, Boehringer-Ingelheim, and Ono. C.S. has consulted for Pfizer, Novartis, GlaxoSmithKline, MSD, BMS, Celgene, AstraZeneca, Illumina, Genentech, Roche-Ventana, GRAIL, Medicxi, and the Sarah Cannon Research Institute. C.S. is a shareholder of Apogen Biotechnologies, Epic Bioscience, GRAIL, and has stock options in and is co-founder of Achilles Therapeutics. E.S. receives research support from AstraZeneca, Glaxo SmithKline, Merck Sharp \& Dhome and is on the advisory board of Phenomic AI. 
2 Figure 1. Construction of in silico tumours.

3 (a) Schematic figure illustrating future evolutionary trajectories delineated by present under4 detected subclones.

5 (b) Schematic figure of probabilistic growth, death, and driver acquisition in a coarse-grained 6 cellular automaton model.

7 (c) Schematic figure of two growth modes: "Surface Growth" with proliferation predominating 8 at the tumour surface and "Volume Growth" with proliferation throughout the tumour volume.

9 (d) Schematic figure of three levels of measurements: from three-dimensional (3D) tumour to 10 two-dimensional (2D) tumour slice and 2D tumour regions within the slice.

11 (e) Representative in-silico tumours under Volume Growth (i) and Surface Growth (ii), 12 respectively, from a 3D view. Tumour voxels harbouring select drivers, as indicated in the 13 figure, are colour coded. Tumour voxels harbouring gain of chromosome arm events are in 14 green; tumour voxels harbouring loss of chromosome arm events are in red. Different shades of greens or reds are employed to reflect different driver events.

17 Figure 2. Clonal diversity in the whole tumour.

18 (a) Schematic figure for the whole-tumour analysis of clonal diversity.

19 (b) Heatmap showing the average number of clones (i.e., parental clone and subclones) with respect to driver acquisition probability and proliferative advantage in the Volume Growth (i) and Surface Growth (ii) models. The average is calculated from 50 in silico tumours per parameter condition. Clones with a whole-tumour cancer cell fraction (CCF) of at least 0.05 are counted.

(c) Whole-tumour CCF of parental and largest subclones in in silico tumours under Volume Growth (i-ii) and Surface Growth (iii), respectively. Driver acquisition probabilities in these sets of simulations are $p_{\text {driver }}=2 \times 10^{-4}$ in (i), $1 \times 10^{-3}$ in (ii), $2 \times 10^{-4}$ in (iii), respectively. "Parental ( $3 p$ loss, $V H L$ )" clone is shown along with up to five subclones with a whole-tumour CCF of 0.01 or higher. All remaining subclones are represented in the "other" group.

(d) Whole-tumour CCF of parental subclones in in silico tumours under Volume Growth and Surface Growth with varying driver acquisition probabilities. N = 100 for each condition.

(e) Shannon diversity index in in silico tumours under Volume Growth and Surface Growth with varying driver acquisition probabilities. $\mathrm{N}=100$ for each condition.

Statistical annotations in (d-e) reflect two-sided Wilcoxon tests: “****” indicates $P \leq 0.0001$.

Figure 3. Between-region genomic divergence.

(a) Schematic figure and procedure for the analysis in 2D tumour regions.

(b) Spatial locations of regional samples with tumour edge indicated in a representative in silico tumour under Surface Growth.

(c) Between-region spatial distance against genomic distance derived from the representative in silico tumour in panel b. Overlapping data points are counted (" $n$ "). Pearson correlation (" $r$ ") and $p$ value are indicated in the figure.

(d-e) The same analysis as described in (b-c) for a representative in silico tumour with Volume Growth.

(f) Pearson correlation between spatial distance and genomic distance in in silico tumours under Volume Growth and Surface Growth with varying driver acquisition probabilities. $\mathrm{N}=$ 100 for each condition. 
1 Statistical annotations in (f) reflect two-sided Wilcoxon tests: “****" indicates $P \leq 0.0001$

2 and "ns" indicates no statistical significance.

3

4

Figure 4. Spatial features of clonal diversity.

(a) Schematic figure and procedure for the analysis within a 2D tumour.

(b) Spatial maps of subclones (i) and microdiversity (ii) in a representative in-silico tumour under Surface Growth.

(c) The same analysis as described in (b) for a representative in-silico tumour under Volume Growth.

(d) Maps of regional biopsies with the number of subclones within a biopsy colour coded in two cases (G_K234 and G_K446) in the TRACERx Renal study. Hues from red to purple to blue reflect decreasing number of subclones. "Low" reflect zero subclones, while "High" reflects the maximum number of subclones found in any region (i.e., 4 subclones in G_K234, 3 subclones in G_K446).

(e) Cumulative probability distribution, $P(D \leq d)$, of the normalised distance to tumour centre in in silico tumours under Surface Growth and Volume Growth and in ccRCC tumours. Three sets of in-silico tumours with different driver acquisition probabilities are shown. $\mathrm{N}=100$ for each model condition. "S" and "V" in the figure reflect Surface Growth and Volume Growth, respectively. " $p=2 \mathrm{e}-4$ " reflects a driver acquisition probability of $2 \mathrm{e}-4.606$ patient tumour (PT) regions from $54 \mathrm{ccRCC}$ tumours are considered for the experimental analysis.

(f) Bootstrapped power law exponent $k$, as in $P(D \leq d) \sim d^{k}$, fitted to cumulative probability distribution of normalised distance to tumour centre in each of bootstrap samples. For microdiversity hotspots under each model condition, 100 bootstrap samples are generated by randomly sampling 400 hotspots with replacement.

(g) Bootstrapped power law exponent $k$ in ccRCC tumours.

Driver acquisition probabilities used in representative simulations are: $p_{\text {driver }}=2 \times 10^{-4}$ in both in-silico tumours under Surface Growth (b) and in-silico tumours under Volume Growth (c).

Statistical annotations in (f) reflect two-sided Wilcoxon tests: “****” indicates $P \leq 0.0001$.

Figure 5. Frequency and spatial features of parallel evolution.

(a) Schematic figure for measuring parallel evolution in the 3D tumour (i) and for analysing parallel mutational events within 2D tumour regions (ii).

(b) Heatmap showing the fraction of in silico tumours that have parallel evolution in PBRMI with respect to driver acquisition probability and growth advantage in the Volume Growth (i) and Surface Growth (ii) models. The fraction is calculated based on 50 in silico tumours per parameter condition. In silico tumours are counted, if there are two or more parallel mutational events in $P B R M 1$ with a whole-tumour CCF of at least 0.05 .

(c-d) Spatial distribution of PBRM1 or BAPI mutation (i) and its parallel events (ii) in a representative in silico tumour under Surface Growth (c) and Volume Growth (d), respectively. In panel (ii), different colours overlaid with "PBRMI" or "BAPI" reflect different parallel mutation events.

(e) Maximum distances to the tumour edge from parallel mutations in PBRM1 or BAP1 that span up to two regions in in silico tumours under Volume Growth and Surface Growth with varying driver acquisition probabilities. $\mathrm{N}=100$ for each condition.

(f) Maps of regions containing parallel mutations in PBRM1 in a representative case (G_K520) in the TRACERx Renal study. Distinct parallel mutations are coloured differently. For regions containing more than two parallel mutations, two colours are applied simultaneously. Doubleheaded arrow indicates a measurement of distance to tumour edge in this example. 
1 (g) Maximum distances from mutational events that span up to two regions in ccRCC tumours 2 in the TRACERx Renal study. For comparison, all mutations ("All mutations"), mutations in 3 driver genes ("Drivers"), and parallel mutations in driver genes ("Drivers (P.E.)") are shown 4 as separate groups.

5 Driver acquisition probabilities used in representative simulations are: $p_{\text {driver }}=2 \times 10^{-4}$ in 6 in silico tumours under Surface Growth and $p_{\text {driver }}=1 \times 10^{-3}$ in in silico tumours under 7 Volume Growth.

8 Statistical annotations in (e) reflect two-sided Wilcoxon tests: “**” indicates $P \leq 0.01$, “*” 9 indicates $P \leq 0.05$, and "ns" indicates no statistical significance.

Figure 6. Early predictive indicators of evolutionary trajectories.

(a) The number of subclones as a function of the diameter of a $2 \mathrm{D}$ tumour slice in in silico tumours under Surface Growth and under Volume Growth, respectively. N = 50 simulations with $p_{\text {driver }}=6 \times 10^{-4}$ are shown for each condition.

(b) Kernel density estimation (KDE) with respect to the number of subclones and the diameter of a 2D tumour slice in in silico tumours under Volume Growth (i) and under Surface Growth (ii). Each KDE plot is based on 250 simulations (50 per condition) under 5 conditions with $p_{\text {driver }}=2 \times 10^{-4}, 4 \times 10^{-4}, 6 \times 10^{-4}, 8 \times 10^{-4}, 1 \times 10^{-3}$.

(c) The spatial patterns of parallel mutations in $B A P 1$ over time in a representative in silico tumour under Surface Growth. The red arrow indicates a budding structure at early stage of subclonal expansion.

(d) The CCFs of $B A P 1$ clones within the 2D tumour slice as a function of the diameter of a 2D tumour slice in each of 50 in silico tumours. Only BAPl clones with an ultimate CCF of 0.01 or higher are shown. For those with an ultimate CCF of 0.1 or higher, the diameters of tumour slices at which these clones first became detectable are recorded, with the median indicated by the dashed line. The others with an ultimate CCF of 0.1 or lower are shown in grey.

(e) Axial image in the corticomedullary contrast phase of a representative case (G_K523) showing budding structure on the tumour surface (red arrow). Outlines in red were drawn giving volumetric tumour coverage by an oncologist (S.S.) and a radiologist (D.A.) (f) Maps of tumour regions with the number of subclones colour coded in a representative case (G_K523). Hues from red to purple to blue reflect decreasing number of subclones. "Low" reflect zero subclones, while "High" reflects the maximum number of subclones found in any region (i.e., 4 subclones in G_K523).

(g) The number of subclones as a function of ultimate tumour size in the TRACERx Renal study, overlaid with kernel density estimation based on simulated data. Tumours with a size smaller than $7 \mathrm{~cm}$ and with radiologically evident budding structures on the tumour surface are highlighted (orange). Contours reflect $90 \%$ probability density based on in silico tumours under Surface Growth (red) and under Volume Growth (blue), respective in Figure 6b. 
3

1. Merlo LM, Pepper JW, Reid BJ, Maley CC. Cancer as an evolutionary and ecological process. Nat Rev Cancer. 2006;6(12):924-935. doi:10.1038/nrc2013

2. Zahir N, Sun R, Gallahan D, Gatenby RA, Curtis C. Characterizing the ecological and evolutionary dynamics of cancer. Nat Genet. 2020;52(8):759-767. doi:10.1038/s41588-020-0668-4

3. McGranahan N, Swanton C. Clonal Heterogeneity and Tumor Evolution: Past, Present, and the Future. Cell. 2017;168(4):613-628. doi:10.1016/j.cell.2017.01.018

4. Gerlinger M, Rowan AJ, Horswell S, et al. Intratumor heterogeneity and branched evolution revealed by multiregion sequencing [published correction appears in N Engl J Med. 2012 Sep 6;367(10):976]. N Engl J Med. 2012;366(10):883-892. doi:10.1056/NEJMoa1113205

5. Yates, L., Gerstung, M., Knappskog, S. et al. Subclonal diversification of primary breast cancer revealed by multiregion sequencing. Nat Med 21, 751-759 (2015). https://doi.org/10.1038/nm.3886

6. Jamal-Hanjani M, Wilson GA, McGranahan N, et al. Tracking the Evolution of NonSmall-Cell Lung Cancer. N Engl J Med. 2017;376(22):2109-2121. doi:10.1056/NEJMoa1616288

7. Turajlic S, Xu H, Litchfield K, et al. Deterministic Evolutionary Trajectories Influence Primary Tumor Growth: TRACERx Renal. Cell. 2018;173(3):595-610.e11.

8. McGranahan N, Swanton C. Biological and therapeutic impact of intratumor heterogeneity in cancer evolution [published correction appears in Cancer Cell. 2015 Jul 13;28(1):141]. Cancer Cell. 2015;27(1):15-26. doi:10.1016/j.ccell.2014.12.001

9. Lopez JI, Cortes JM. A divide-and-conquer strategy in tumor sampling enhances detection of intratumor heterogeneity in routine pathology: A modeling approach in clear cell renal cell carcinoma. F1000Res. 2016;5:385. Published 2016 Mar 22. doi:10.12688/f1000research.8196.2

10. Mengelbier LH, Karlsson J, Lindgren D, et al. Intratumoral genome diversity parallels progression and predicts outcome in pediatric cancer. Nat Commun. 2015;6:6125. Published 2015 Jan 27. doi:10.1038/ncomms 7125 
11. Casasent AK, Schalck A, Gao R, et al. Multiclonal Invasion in Breast Tumors Identified by Topographic Single Cell Sequencing. Cell. 2018;172(1-2):205-217.e12. doi:10.1016/j.cell.2017.12.007

12. Melchor L, Brioli A, Wardell CP, et al. Single-cell genetic analysis reveals the composition of initiating clones and phylogenetic patterns of branching and parallel evolution in myeloma. Leukemia. 2014;28(8):1705-1715. doi:10.1038/leu.2014.13

13. Murugaesu N, Wilson GA, Birkbak NJ, et al. Tracking the genomic evolution of esophageal adenocarcinoma through neoadjuvant chemotherapy. Cancer Discov. 2015;5(8):821-831. doi:10.1158/2159-8290.CD-15-0412

14. Voss MH, Hakimi AA, Pham CG, et al. Tumor genetic analyses of patients with metastatic renal cell carcinoma and extended benefit from mTOR inhibitor therapy. Clin Cancer Res. 2014;20(7):1955-1964. doi:10.1158/1078-0432.CCR-132345

15. Juric D, Castel P, Griffith M, et al. Convergent loss of PTEN leads to clinical resistance to a PI(3)K $\alpha$ inhibitor. Nature. 2015;518(7538):240-244. doi:10.1038/nature13948

16. Watkins TBK, Lim EL, Petkovic M, et al. Pervasive chromosomal instability and karyotype order in tumour evolution. Nature. 2020;587(7832):126-132. doi:10.1038/s41586-020-2698-6

17. Zhao Y, Fu X, Lopez J, et al. Selection of metastasis competent subclones in the tumour interior: TRACERx renal. Research Square. 2020. doi:10.21203/rs.3.rs-61979/v1

18. Rodriguez-Brenes IA, Komarova NL, Wodarz D. Tumor growth dynamics: insights into evolutionary processes. Trends Ecol Evol. 2013;28(10):597-604. doi:10.1016/j.tree.2013.05.020

19. Gerlee P. The model muddle: in search of tumor growth laws. Cancer Res. 2013;73(8):2407-2411. doi:10.1158/0008-5472.CAN-12-4355

20. Sun R, Hu Z, Sottoriva A, et al. Between-region genetic divergence reflects the mode and tempo of tumor evolution. Nat Genet. 2017;49(7):1015-1024. doi:10.1038/ng.3891

21. Chkhaidze K, Heide T, Werner B, et al. Spatially constrained tumour growth affects the patterns of clonal selection and neutral drift in cancer genomic data. PLoS Comput Biol. 2019;15(7):e1007243. Published 2019 Jul 29. doi:10.1371/journal.pcbi.1007243

22. Hoefflin R, Lahrmann B, Warsow G, et al. Spatial niche formation but not malignant progression is a driving force for intratumoural heterogeneity. Nat Commun. 2016;7:ncomms11845. Published 2016 Jun 13. doi:10.1038/ncomms11845 
23. Varela I, Tarpey P, Raine K, et al. Exome sequencing identifies frequent mutation of the SWI/SNF complex gene PBRM1 in renal carcinoma [published correction appears in Nature. 2012 Apr 5;484(7392):130]. Nature. 2011;469(7331):539-542. doi:10.1038/nature09639

24. Peng J, Ma J, Li W, et al. Stabilization of MCRS1 by BAP1 prevents chromosome instability in renal cell carcinoma. Cancer Lett. 2015;369(1):167-174. doi:10.1016/j.canlet.2015.08.013

25. Cross W, Kovac M, Mustonen V, et al. The evolutionary landscape of colorectal tumorigenesis. Nat Ecol Evol. 2018;2(10):1661-1672. doi:10.1038/s41559-018-0642$\mathrm{Z}$

26. Zhai W, Lim TK, Zhang T, et al. The spatial organization of intra-tumour heterogeneity and evolutionary trajectories of metastases in hepatocellular carcinoma. Nat Commun. 2017;8:4565. Published 2017 Feb 27. doi:10.1038/ncomms14565

27. Anderson AR, Weaver AM, Cummings PT, Quaranta V. Tumor morphology and phenotypic evolution driven by selective pressure from the microenvironment. Cell. 2006;127(5):905-915. doi:10.1016/j.cell.2006.09.042

28. Waclaw B, Bozic I, Pittman ME, Hruban RH, Vogelstein B, Nowak MA. A spatial model predicts that dispersal and cell turnover limit intratumour heterogeneity. Nature. 2015;525(7568):261-264. doi:10.1038/nature14971

29. Noble R, Burri D, Kather JN, et al. Spatial structure governs the mode of tumour evolution. 2019. doi: https://doi.org/10.1101/586735

30. Driessens G, Beck B, Caauwe A, Simons BD, Blanpain C. Defining the mode of tumour growth by clonal analysis. Nature. 2012;488(7412):527-530. doi:10.1038/nature11344

31. Williams MJ, Werner B, Barnes CP, Graham TA, Sottoriva A. Identification of neutral tumor evolution across cancer types. Nat Genet. 2016;48(3):238-244. doi:10.1038/ng.3489

32. Crawley MJ, Harral JE. Scale dependence in plant biodiversity. Science. 2001;291(5505):864-868. doi:10.1126/science.291.5505.864

33. Horner-Devine MC, Lage M, Hughes JB, Bohannan BJ. A taxa-area relationship for bacteria. Nature. 2004;432(7018):750-753. doi:10.1038/nature03073

34. Zhou J, Kang S, Schadt CW, Garten CT Jr. Spatial scaling of functional gene diversity across various microbial taxa. Proc Natl Acad Sci U S A. 2008;105(22):7768-7773. doi:10.1073/pnas.0709016105 
35. Fiore VF, Krajnc M, Quiroz FG, et al. Mechanics of a multilayer epithelium instruct tumour architecture and function [published correction appears in Nature. $2020 \mathrm{Sep}$ 11;:]. Nature. 2020;585(7825):433-439. doi:10.1038/s41586-020-2695-9

36. Marcus DS, Olsen TR, Ramaratnam M, Buckner RL. The Extensible Neuroimaging Archive Toolkit: an informatics platform for managing, exploring, and sharing neuroimaging data. Neuroinformatics. 2007;5(1):11-34. doi:10.1385/ni:5:1:11 

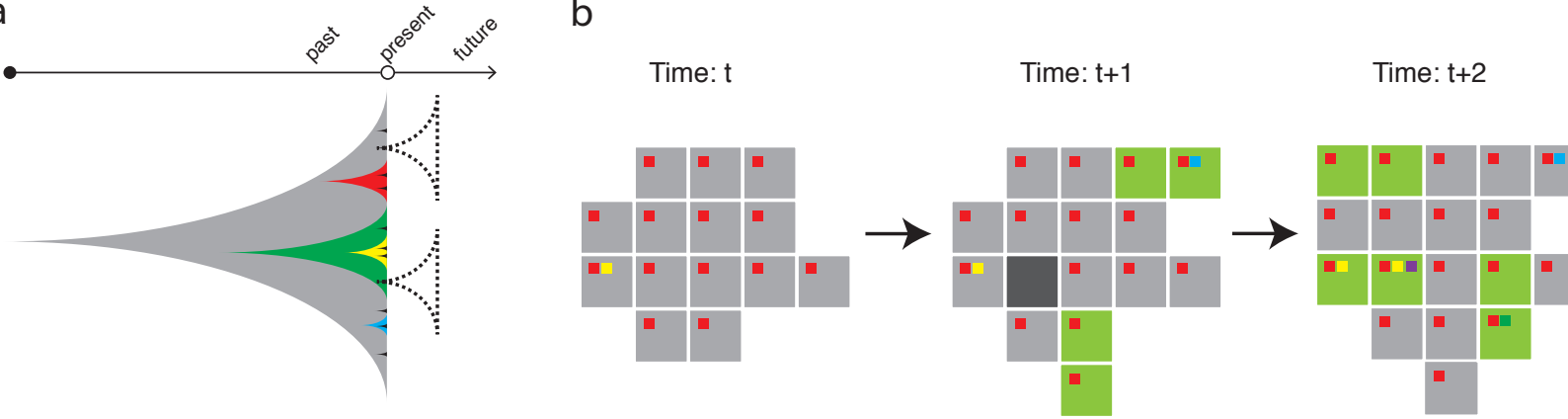

$=\stackrel{p_{\text {growth }}}{\longrightarrow=}$

$\stackrel{p_{\text {death }}}{\longrightarrow}$

$\stackrel{p_{\text {driver }}}{\longrightarrow}=$

\section{$<-<>$ parental clone \\ 4 "micro"-clones \\ evolutionary potential}

Tumour voxel $\square$ New tumour voxel $\square$ Dead tumour voxel $\square=$ Driver mutation or SCNA

C

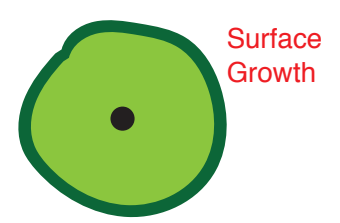

Active growth

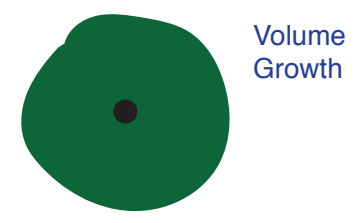

No growth

Tumour origin

d

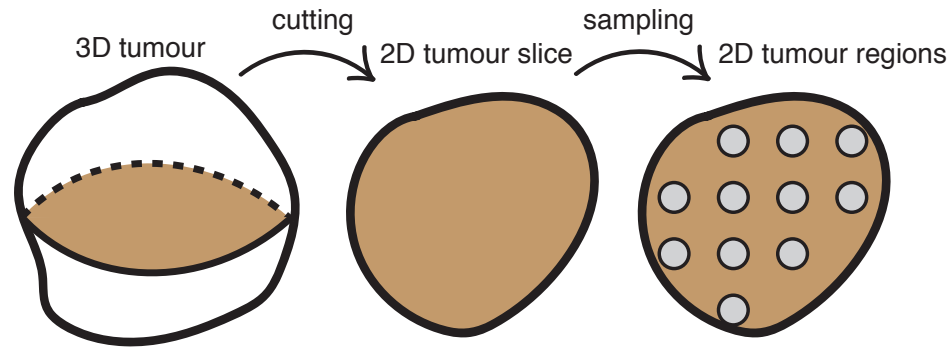

(i) Volume Growth model
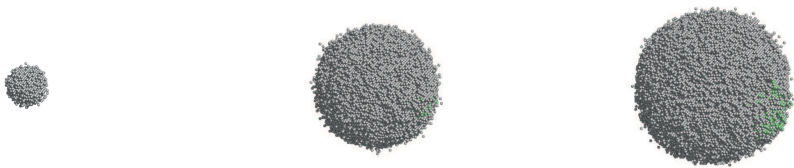

Time

(ii) Surface Growth model

40
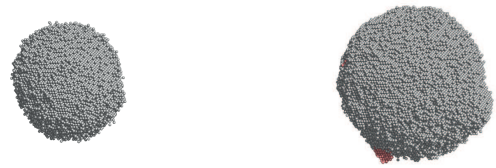

loss_8p
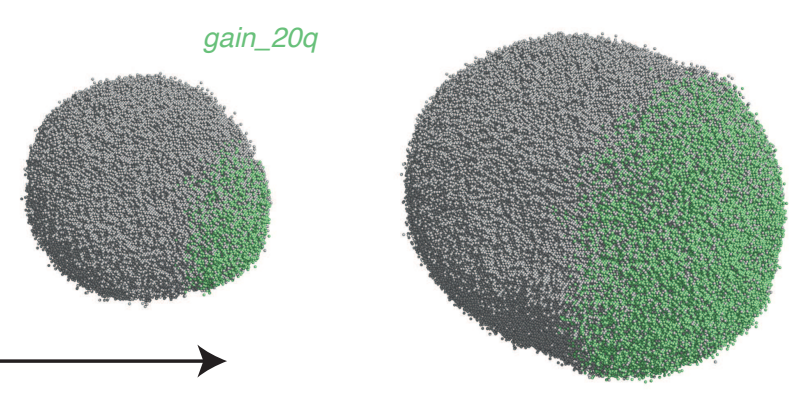

gain_20q

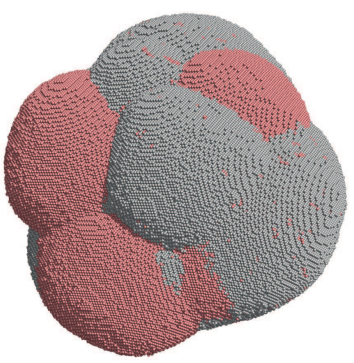

Time

gain_20q
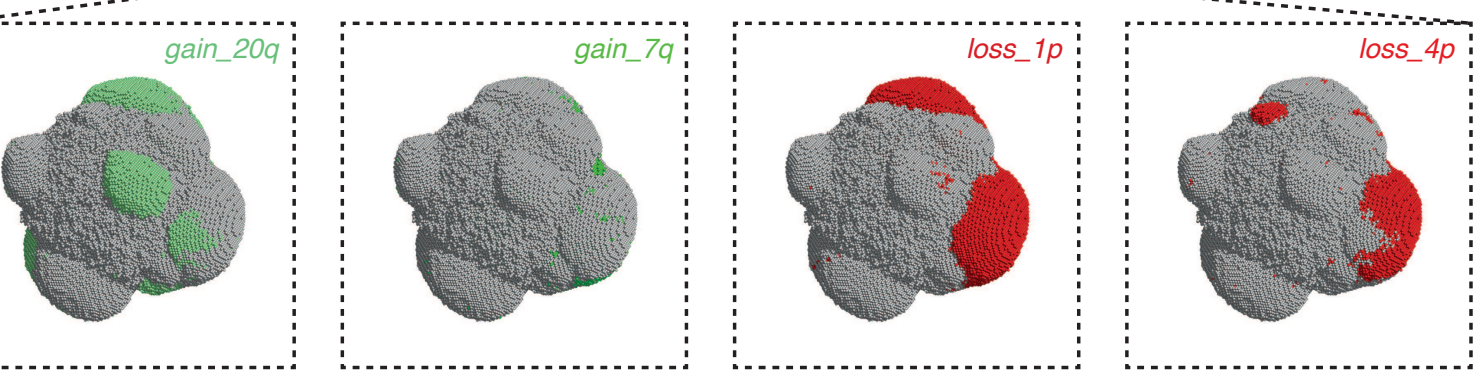


\section{a}

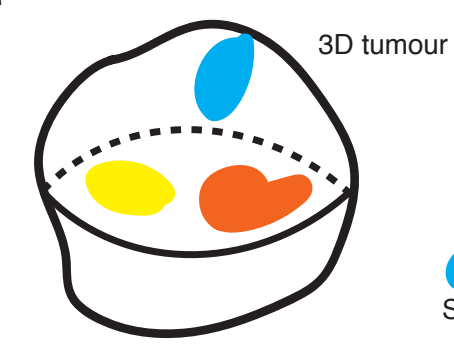

b (i) Volume Growth model

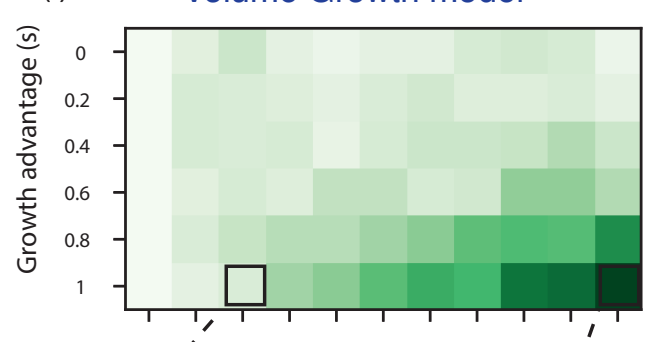

(i) Count subclones in 3D tumour.

(ii) Calculate the whole-tumour CCF of subclones and Shannon diversity. (ii) Surface Growth model

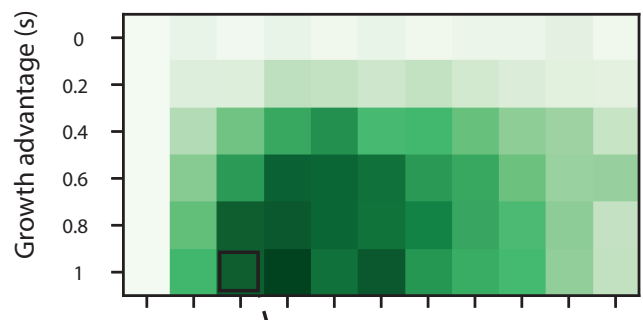

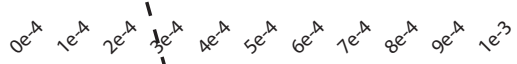
Driver acquisition probability $\left(\mathrm{p}_{\text {driver }}\right)$ 1

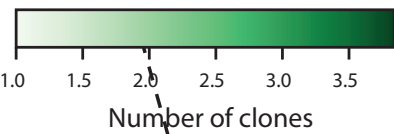
Numper of clones Number of clones,

C Volume Growth model

(i) $p_{\text {driver }}=2 \times 10^{-4}$

$\frac{9}{0}$
$\frac{0}{0}$
$\frac{0}{0}$
$\frac{0}{0}$
$\frac{0}{2}$

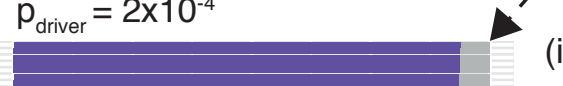

Whole tumour CCF (tumour level)
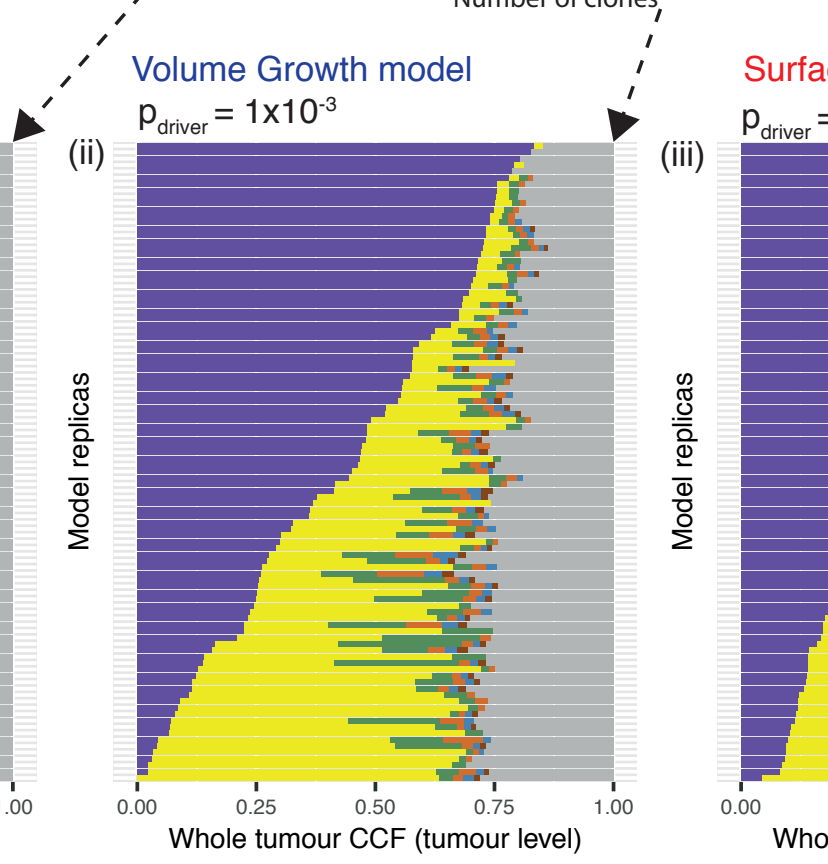

(iii)

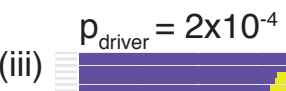

Subclone

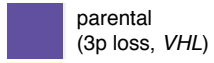

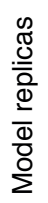

subclone 1

subclone 2

subclone 3

subclone 4

subclone 5

other d

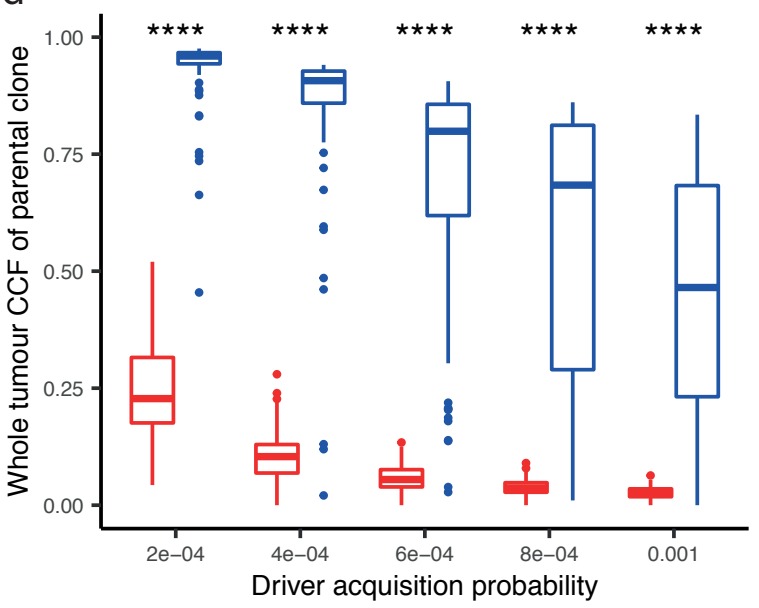

e

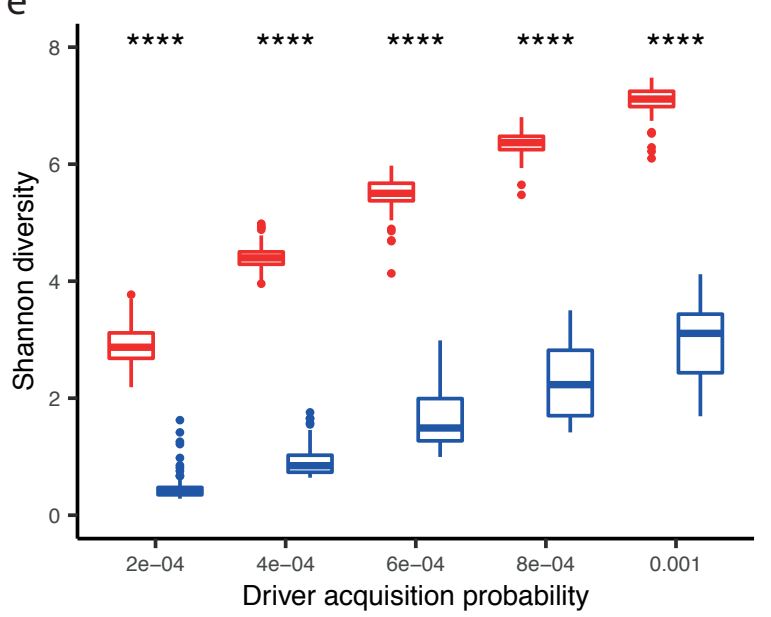

Condition

Surface

官 volume 
$\mathrm{b}$

(i) Sample regions from tumour slice.

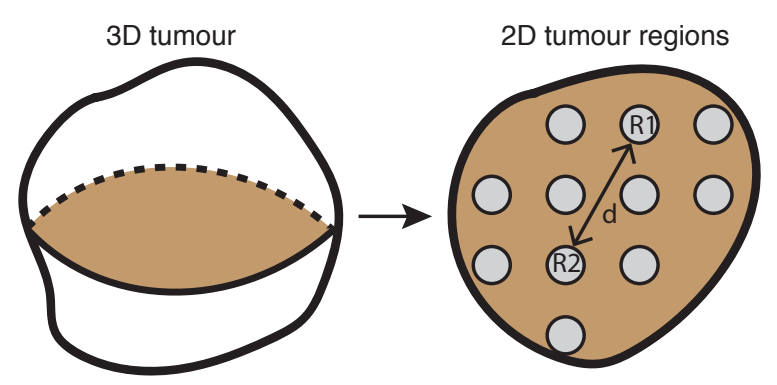

(ii) Collect driver information in each region.

(iii) Calculate spatial and genomic distances between each pair of regions.

(iv) Evaluate correlation between spatial and genomic distances.
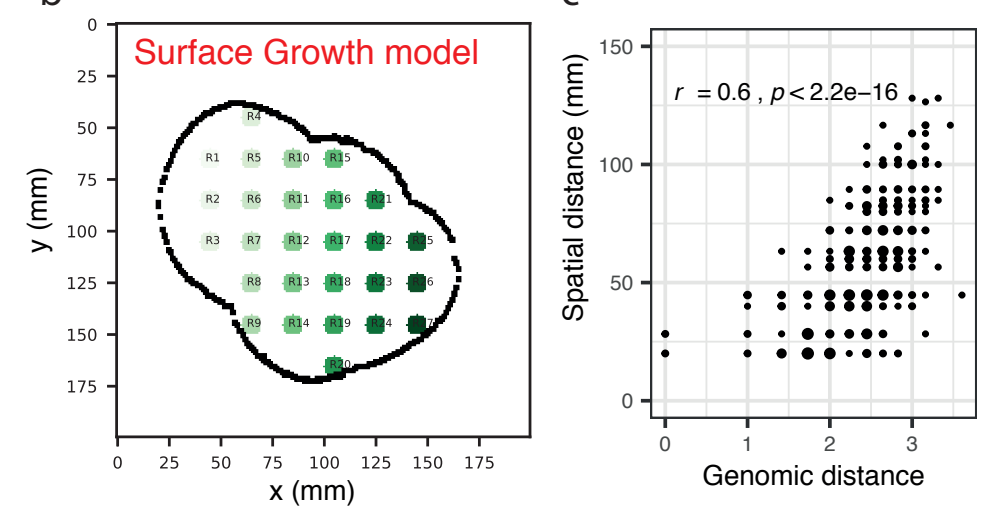

d

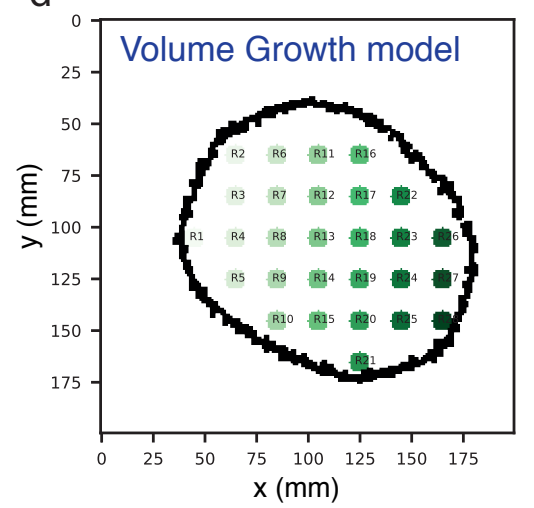

e

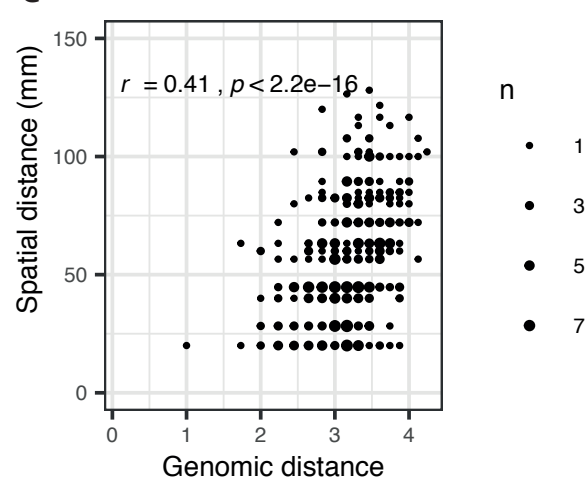

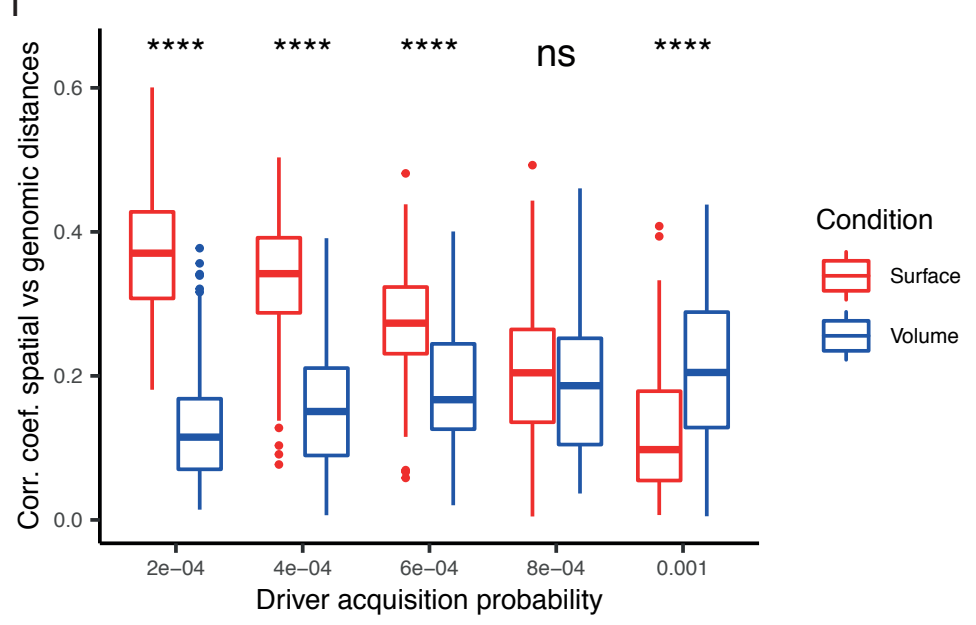




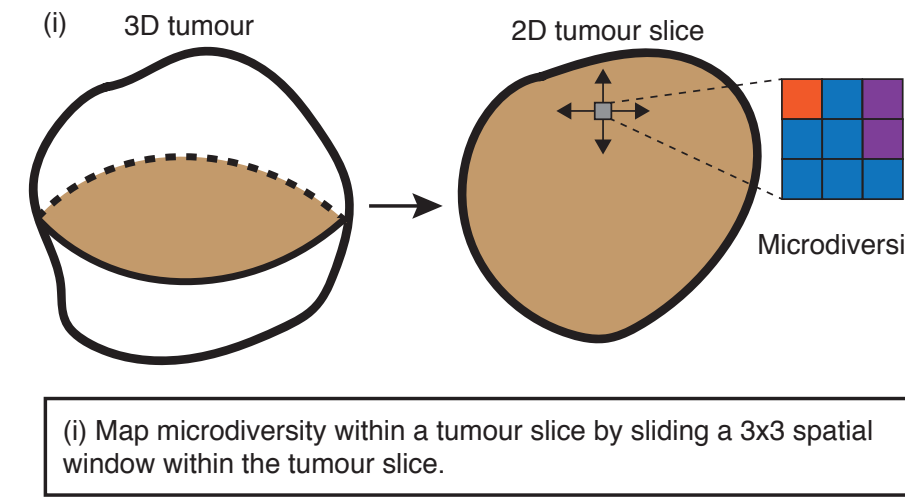

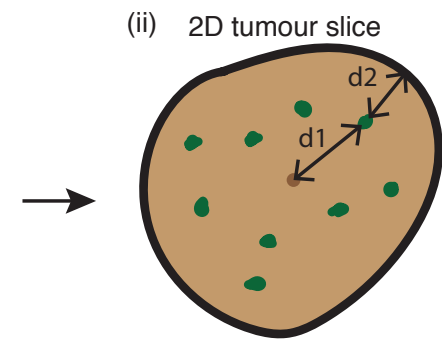

(ii) Evaluate the spatial pattern of microdiversity hotspots within the tumour slice.
Hotspots with microdiversity

of at least 5

- Centre of the tumour slice

d1: distance to tumour centre d2: distance to tumour margin $\mathrm{d}=\mathrm{d} 1 /(\mathrm{d} 1+\mathrm{d} 2)$ :

Normalised distance to tumour centre b (i)

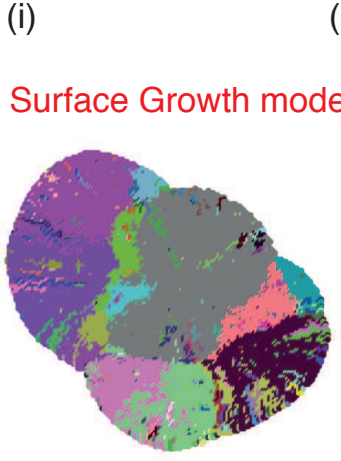

d (i)

(ii)

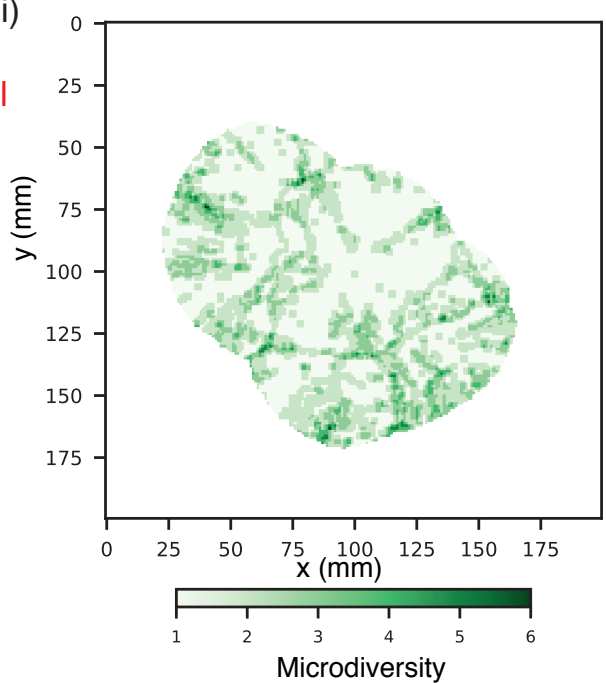

G_K234

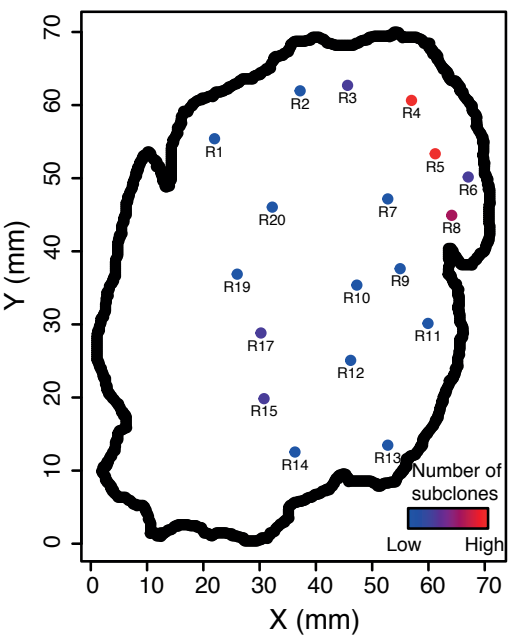

(ii)

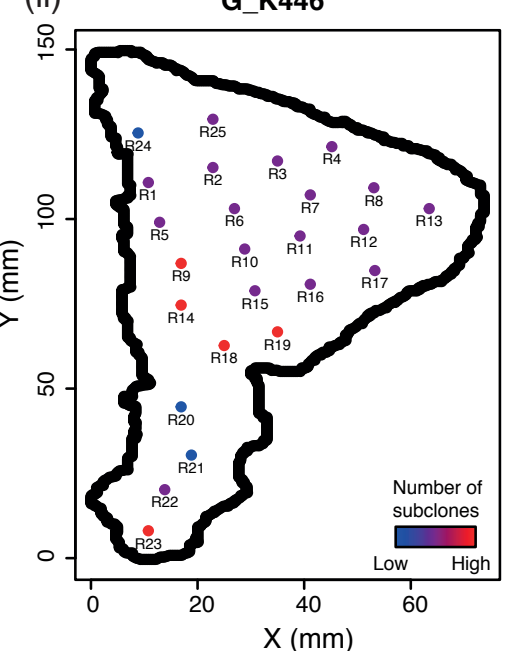

C (i)

Volume Growth model

e

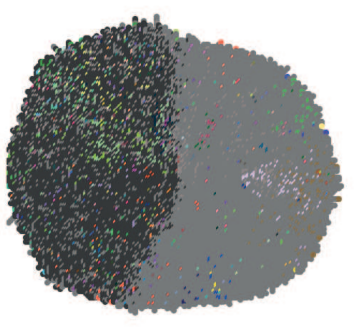

(ii)

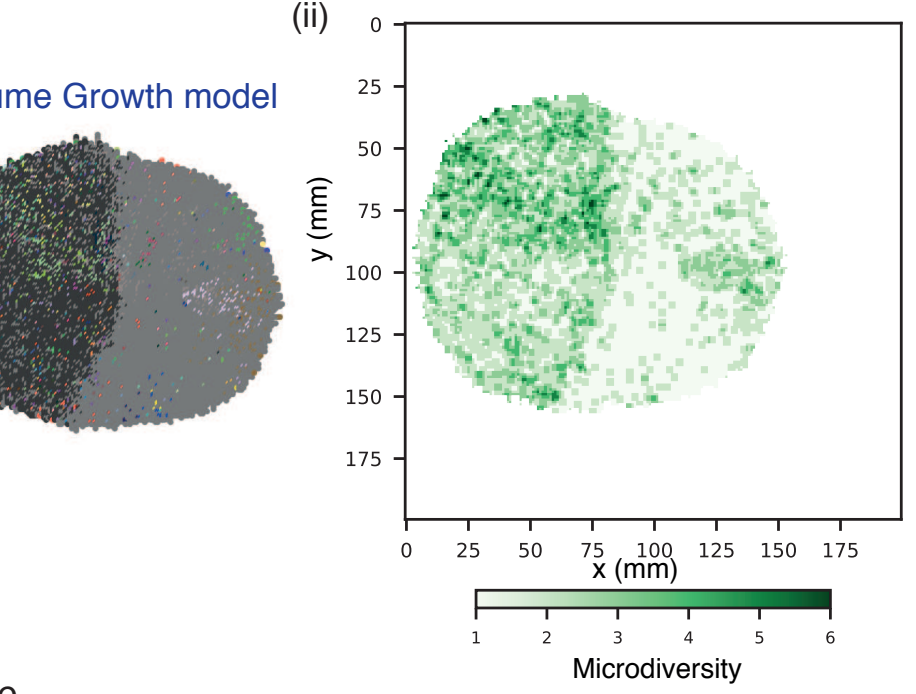

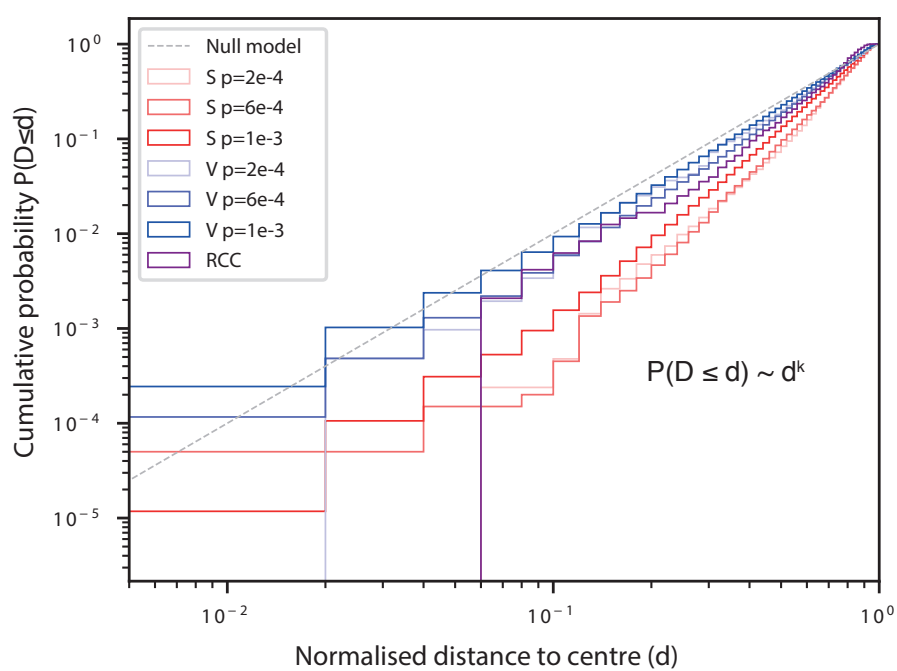

$\mathrm{f}$

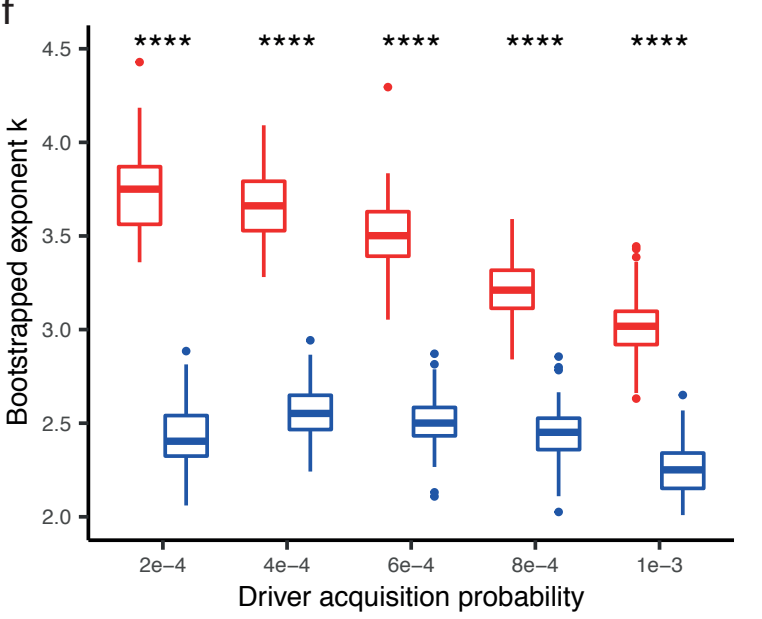

GrowthMode

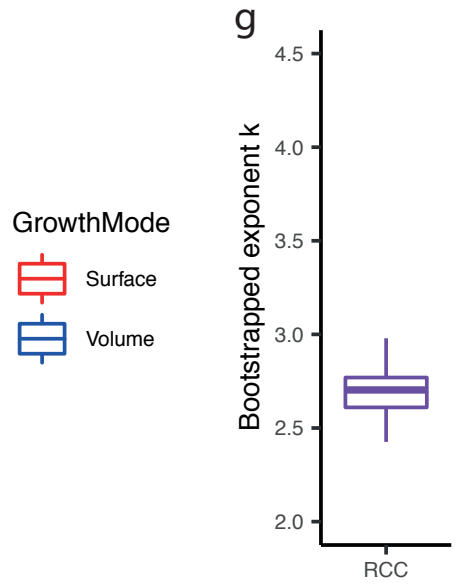

Normalised distance to centre (d) 
$a_{(i)}$
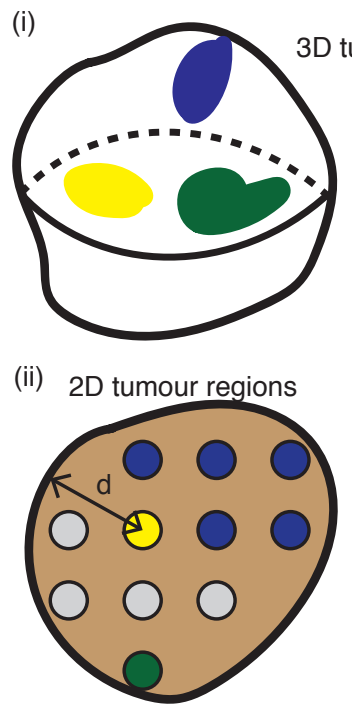

(ii) 2D tumour regions b spanned by parallel mutations in a given driver gene

$\mathrm{d}$ : distance to tumour margin from events with limited clonal expansion
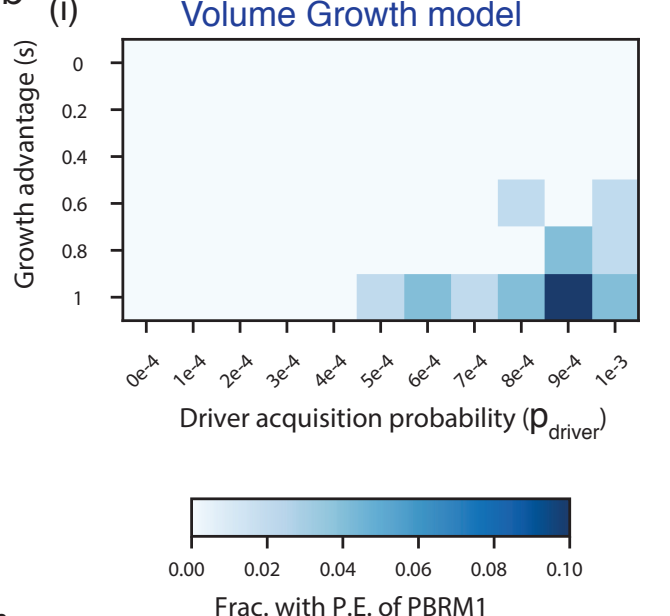

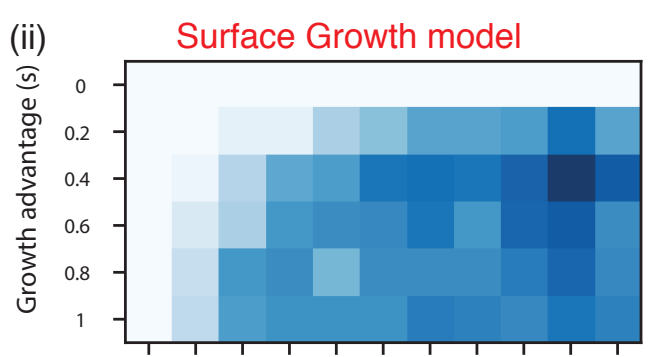

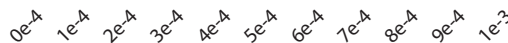
Driver acquisition probability $\left(\mathrm{p}_{\text {driver }}\right)$

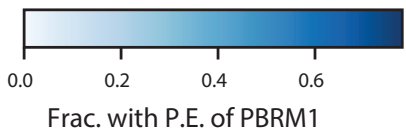

(iii)

d
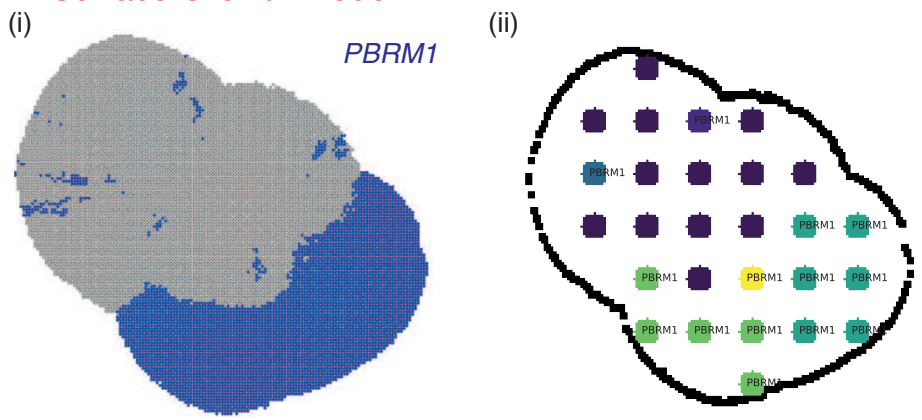

(i)
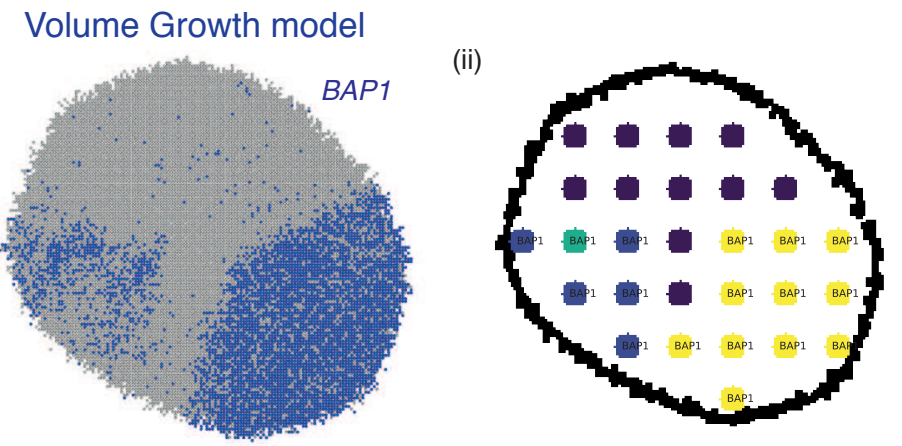

$f$

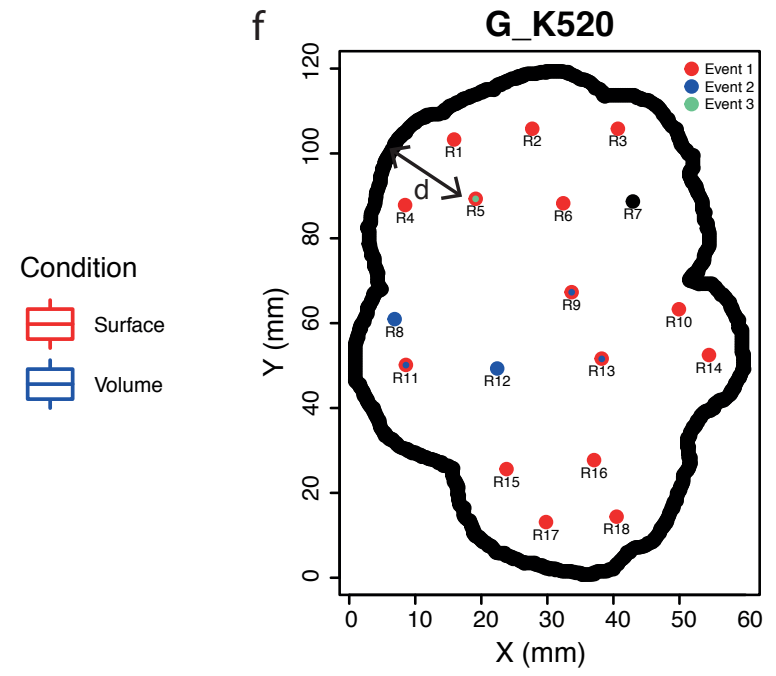

g

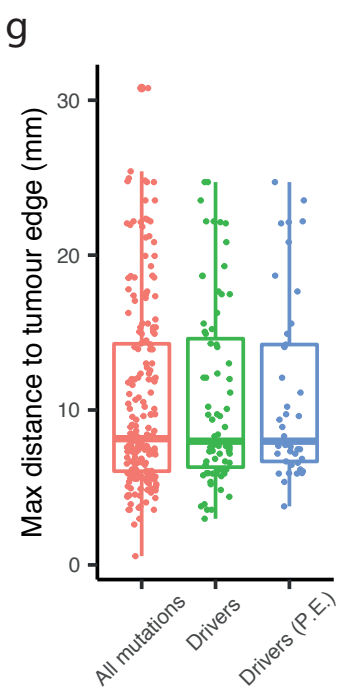

Condition
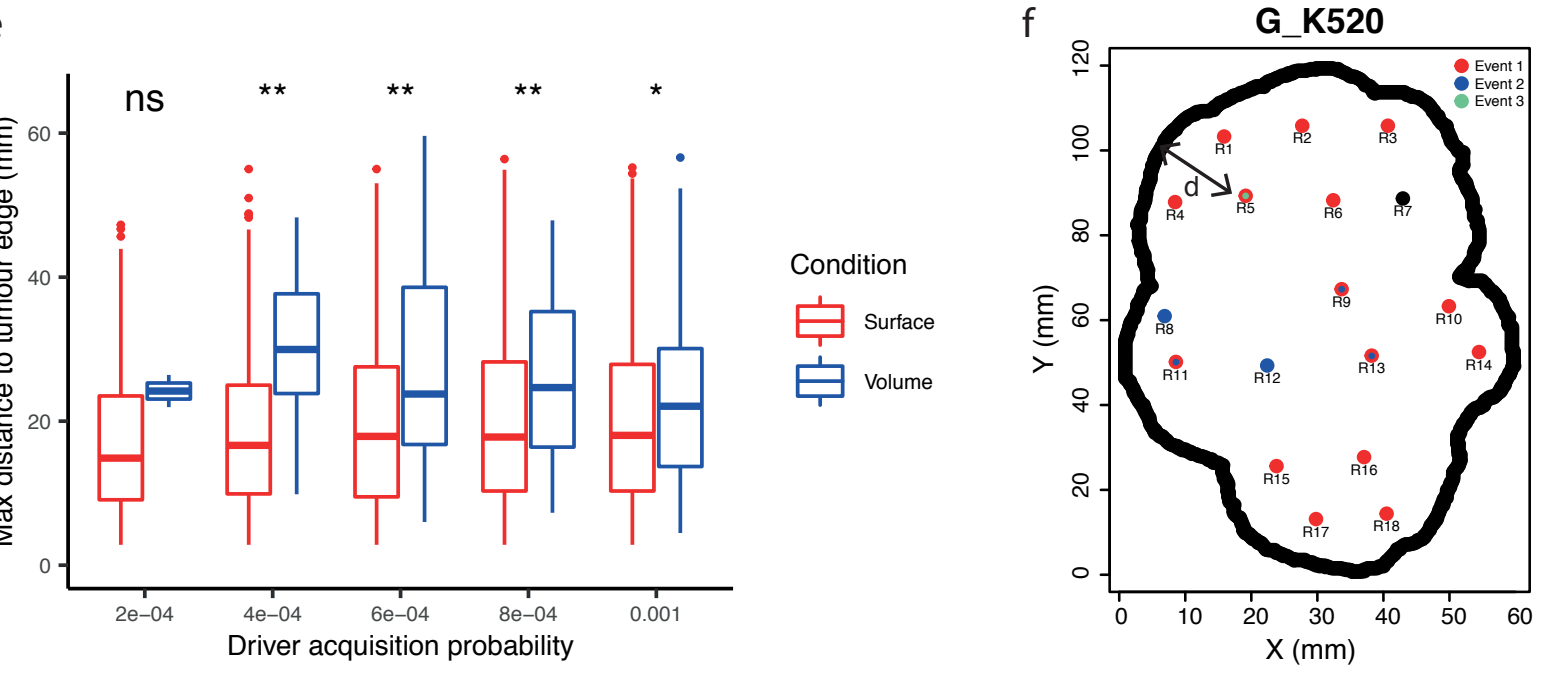

, 
$a$

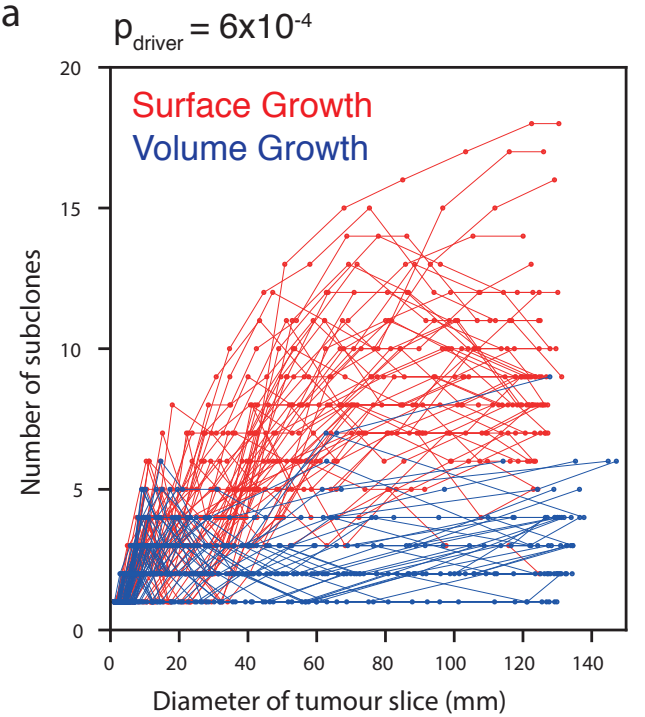

b (i) Volume Growth model

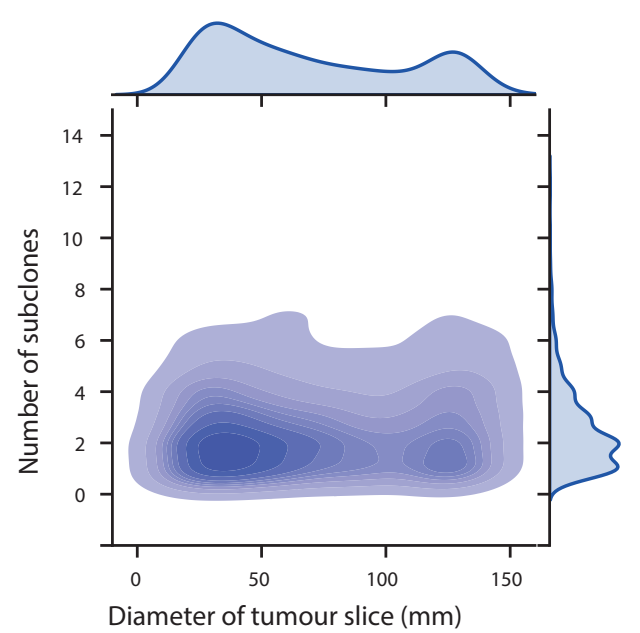

C (i)

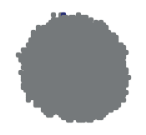

Time

d

(i) $\mathrm{p}_{\text {driver }}=2 \times 10^{-4}$

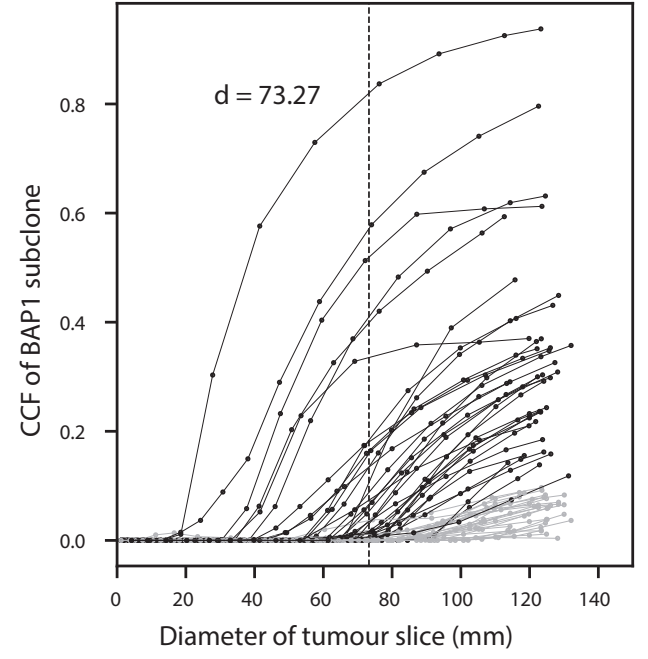

e
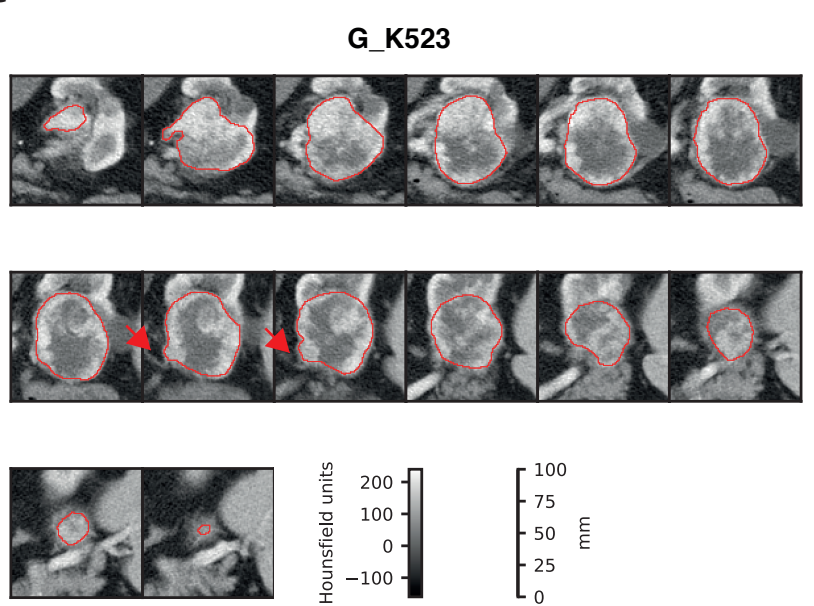

(ii)

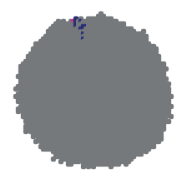

(iii)

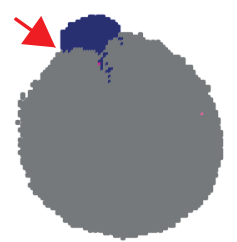

(iv)

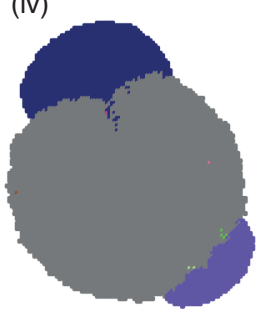

G_K523
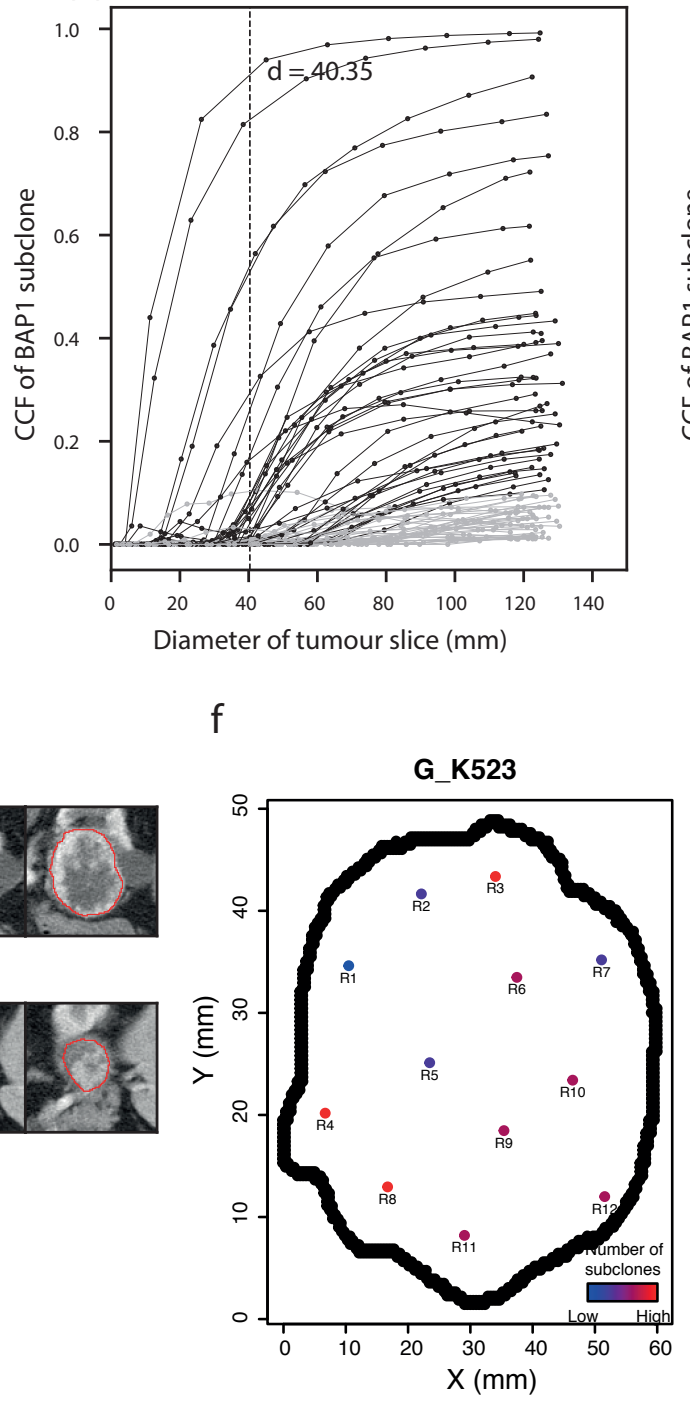

$f$

(v)
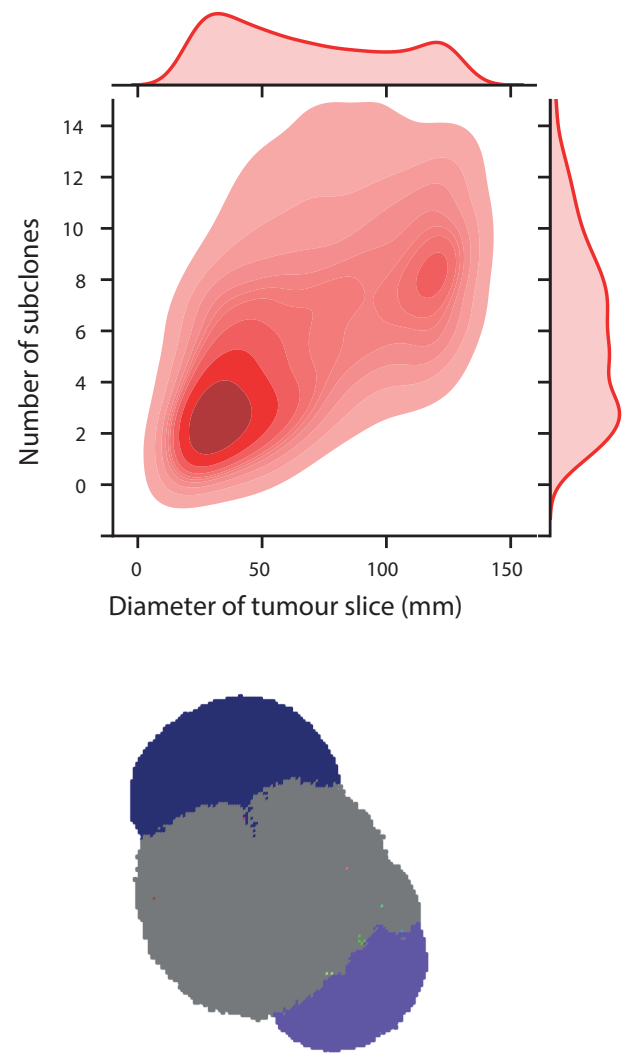

(ii) $\mathrm{p}_{\text {driver }}=1 \times 10^{-3}$

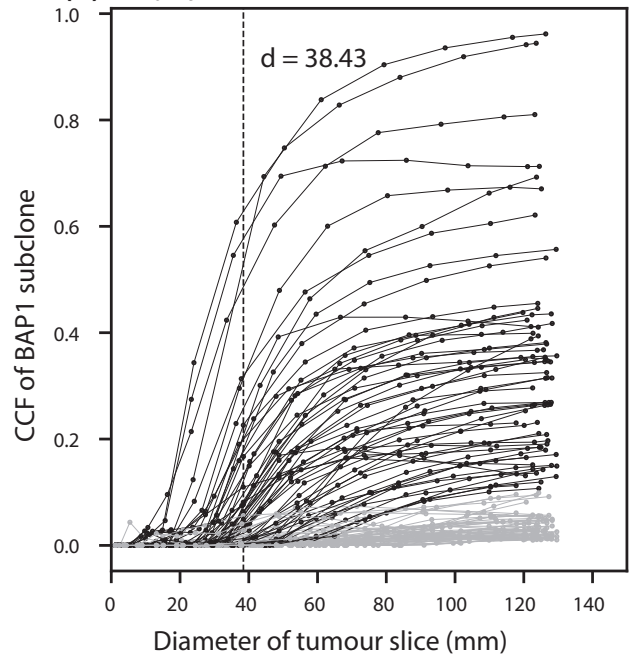

g

(ii) Surface Growth model

Diameter of tumour slice ( $\mathrm{mm}$ )

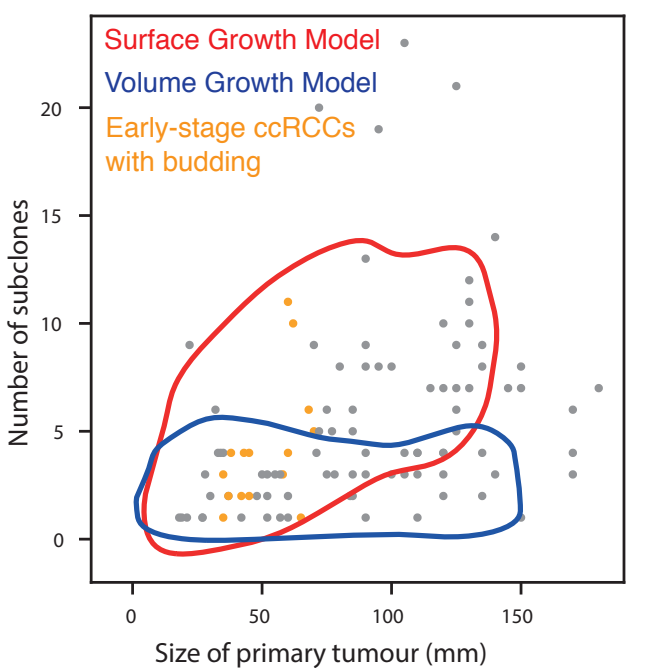



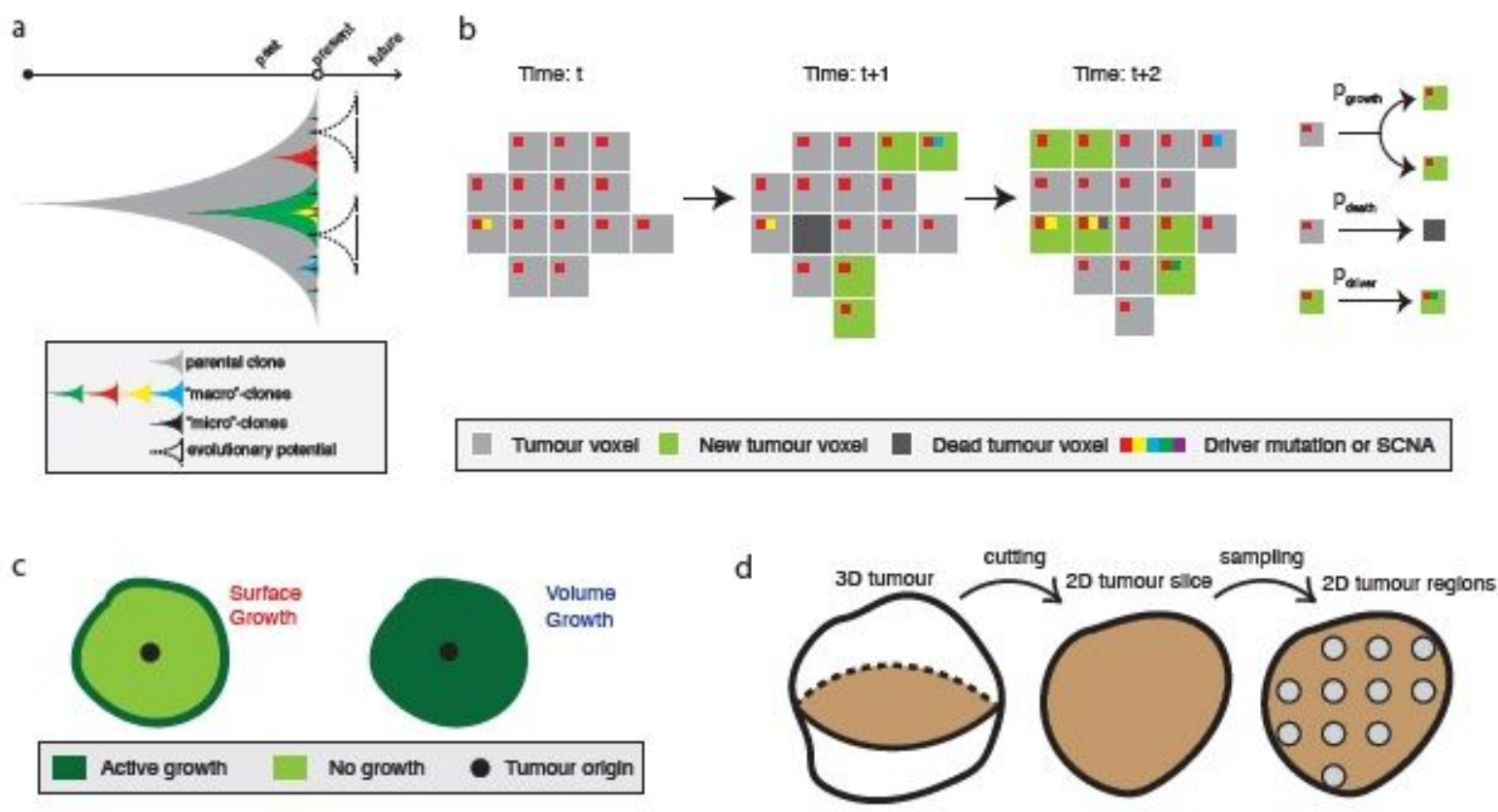

e

(i) Volume Growth model

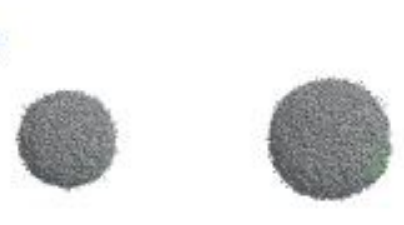
Time

(ii) Surface Growth model
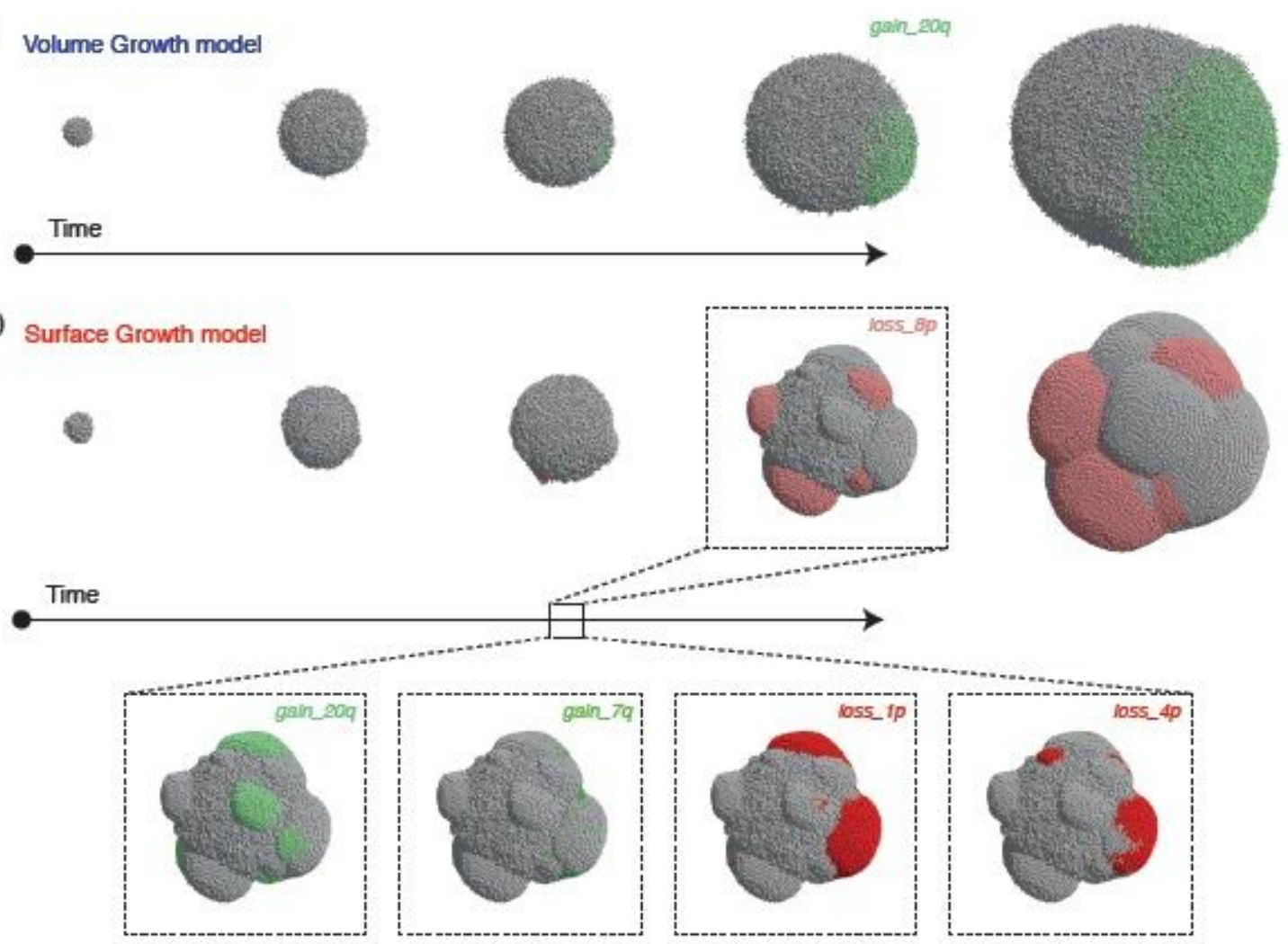

\section{Figure 1}

Construction of in silico tumours. (a) Schematic figure illustrating future evolutionary trajectories delineated by present under detected subclones. (b) Schematic figure of probabilistic growth, death, and driver acquisition in a coarse-grained cellular automaton model. (c) Schematic figure of two growth 
modes: "Surface Growth" with proliferation predominating at the tumour surface and "Volume Growth" with proliferation throughout the tumour volume. (d) Schematic figure of three levels of measurements: from three-dimensional (3D) tumour to two-dimensional (2D) tumour slice and 2D tumour regions within the slice. (e) Representative in-silico tumours under Volume Growth (i) and Surface Growth (ii), respectively, from a 3D view. Tumour voxels harbouring select drivers, as indicated in the figure, are colour coded. Tumour voxels harbouring gain of chromosome arm events are in green; tumour voxels harbouring loss of chromosome arm events are in red. Different shades of greens or reds are employed to reflect different driver events. 
a

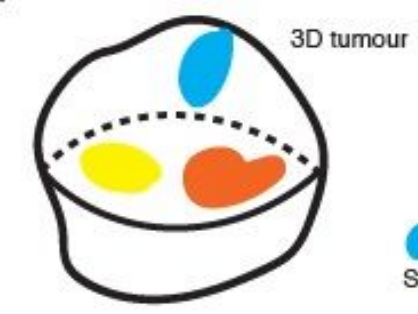

b (i)

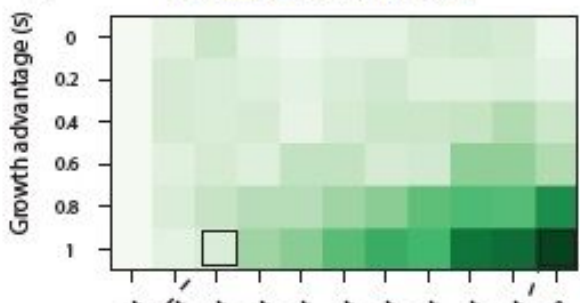

(i) Count subclones in 3D tumour.

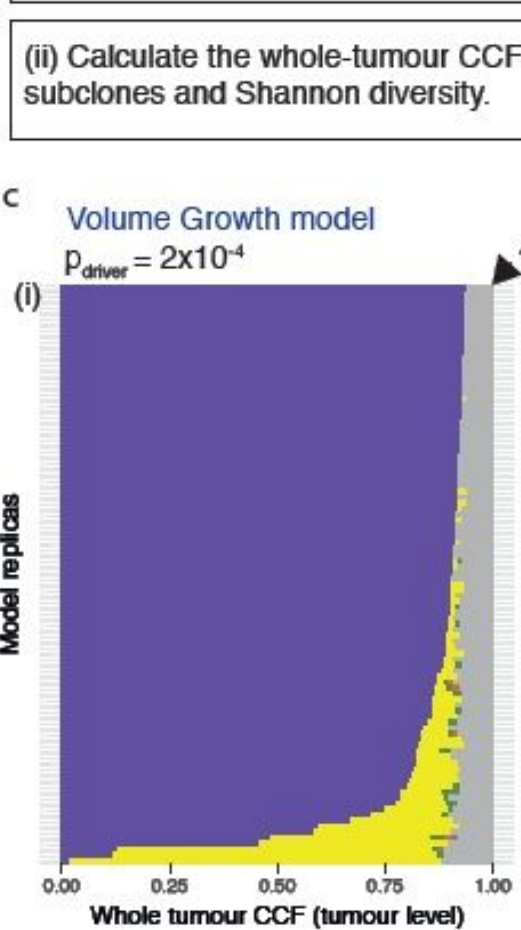

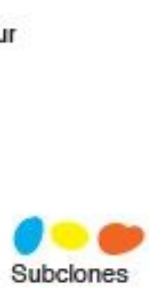

Subclones (ii) Surface Growth model

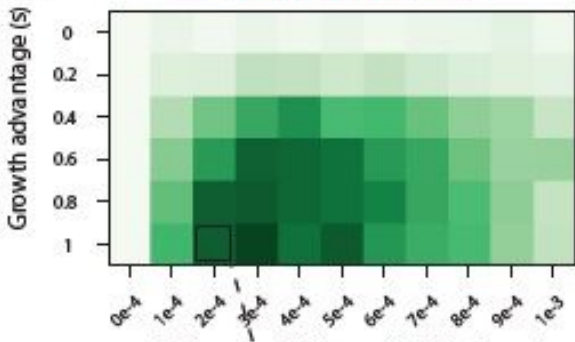

Driver acquisition probability $\left(P_{\text {driver }}\right)$

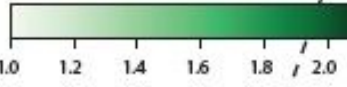

Number of clones'

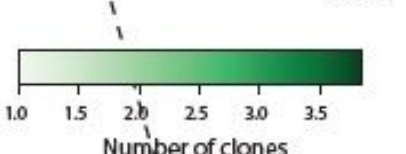

Numper of clones

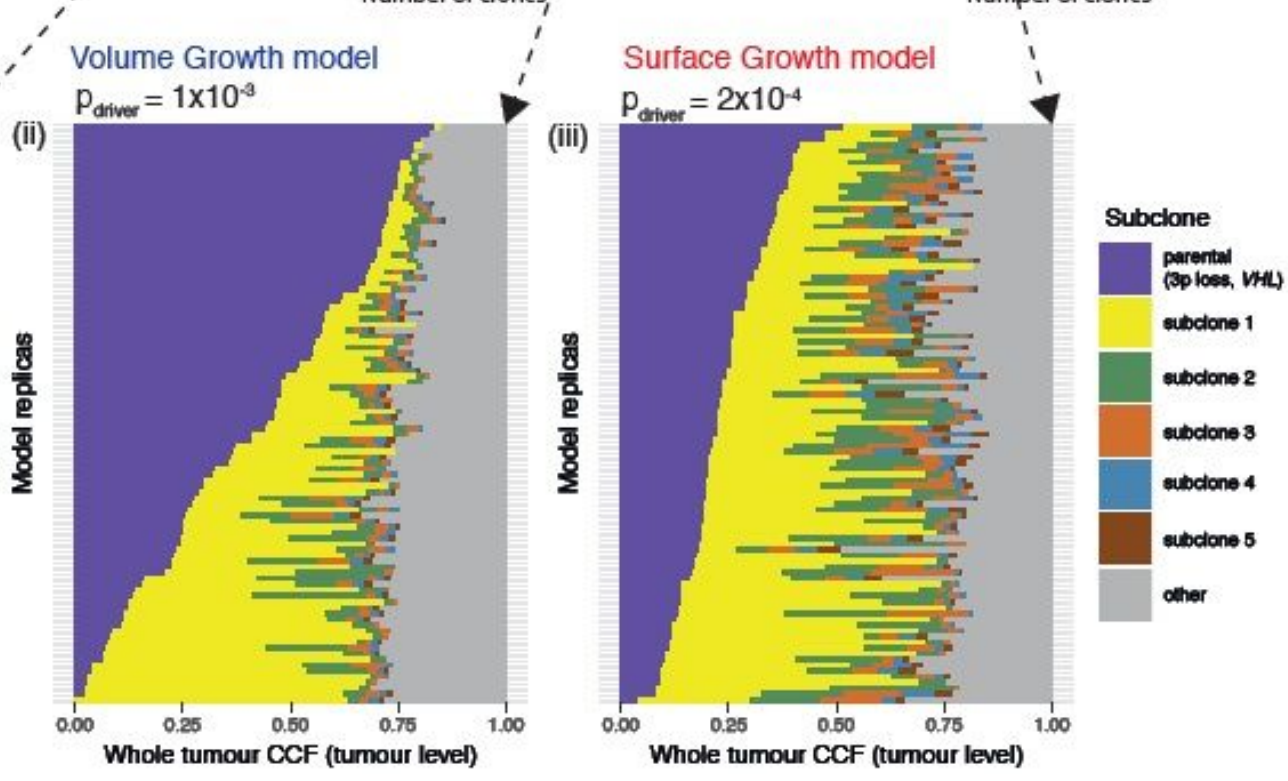

d
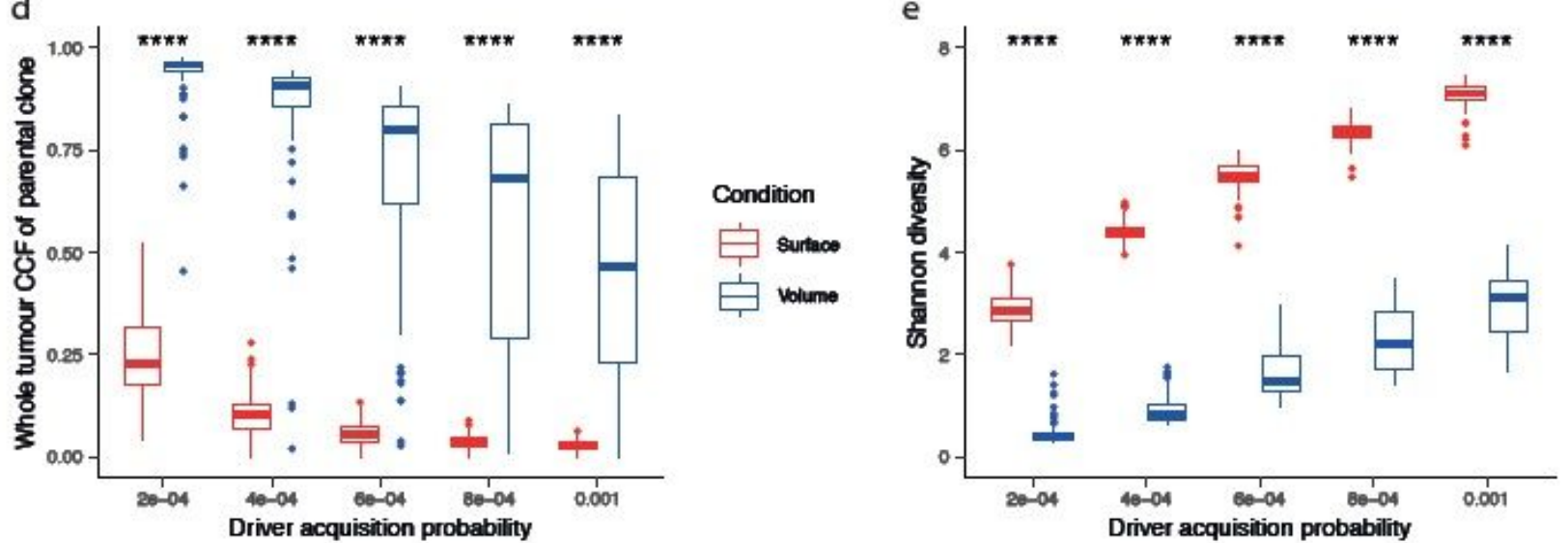

Condition

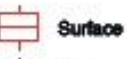

ए volume

Driver acquisition probability

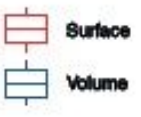

Figure 2

Clonal diversity in the whole tumour. (a) Schematic figure for the whole-tumour analysis of clonal diversity. (b) Heatmap showing the average number of clones (i.e., parental clone and subclones) with respect to driver acquisition probability and proliferative advantage in the Volume Growth (i) and Surface Growth (ii) models. The average is calculated from 50 in silico tumours per parameter condition. Clones with a whole-tumour cancer cell fraction (CCF) of at least 0.05 are counted. (c) Whole-tumour CCF 
of parental and largest subclones in in silico tumours under Volume Growth (i-ii) and Surface Growth (iii), respectively. Driver acquisition probabilities in these sets of simulations are $\$, \#-$./\# = $2 \times 1001$ in (i), $1 \times$ 100 ! in (ii), $2 \times 1001$ in (iii), respectively. "Parental (3p loss, VHL)" clone is shown along with up to five subclones with a whole-tumour CCF of 0.01 or higher. All remaining subclones are represented in the "other" group. (d) Whole-tumour CCF of parental subclones in in silico tumours under Volume Growth and Surface Growth with varying driver acquisition probabilities. N $=100$ for each condition. (e) Shannon diversity index in in silico tumours under Volume Growth and Surface Growth with varying driver acquisition probabilities. $\mathrm{N}=100$ for each condition. Statistical annotations in (d-e) reflect two-sided

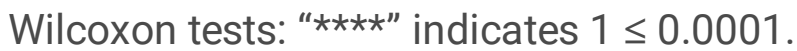

a

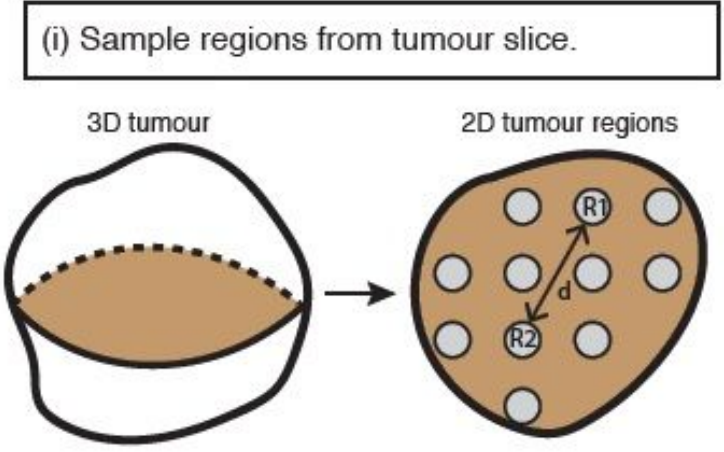

(ii) Collect driver information in each region.

(iii) Calculate spatial and genomic distances between each pair of regions.

(iv) Evaluate correlation between spatial and genomic distances. b

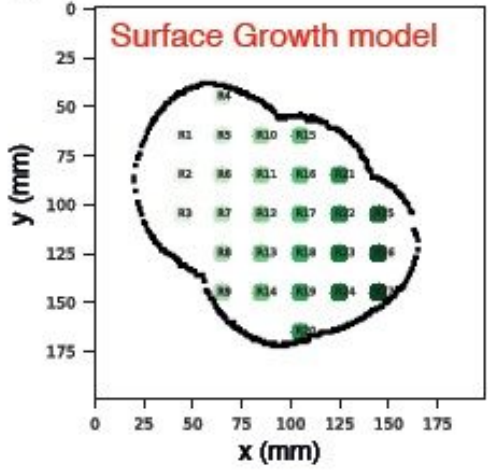

d

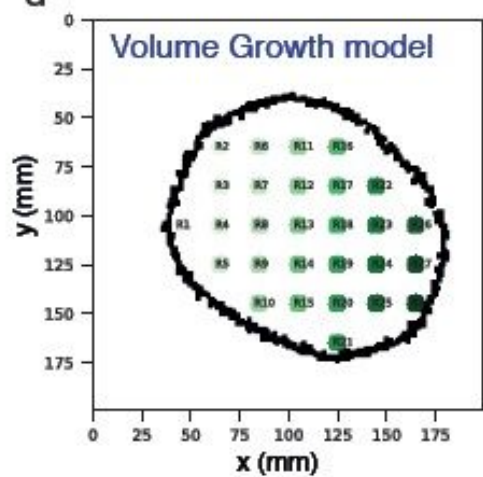

c

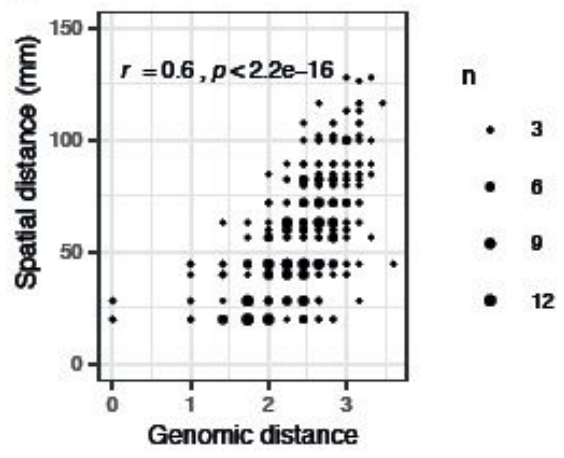

e

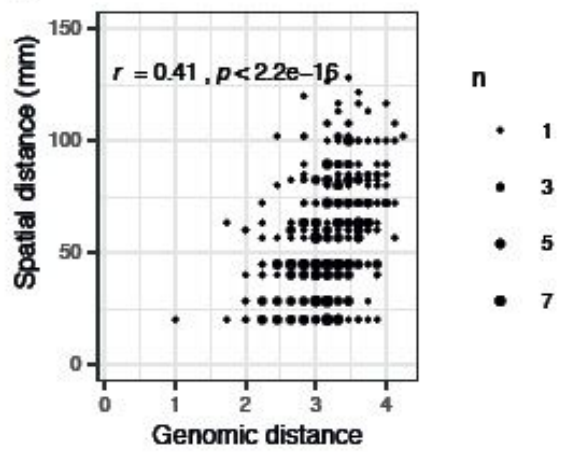

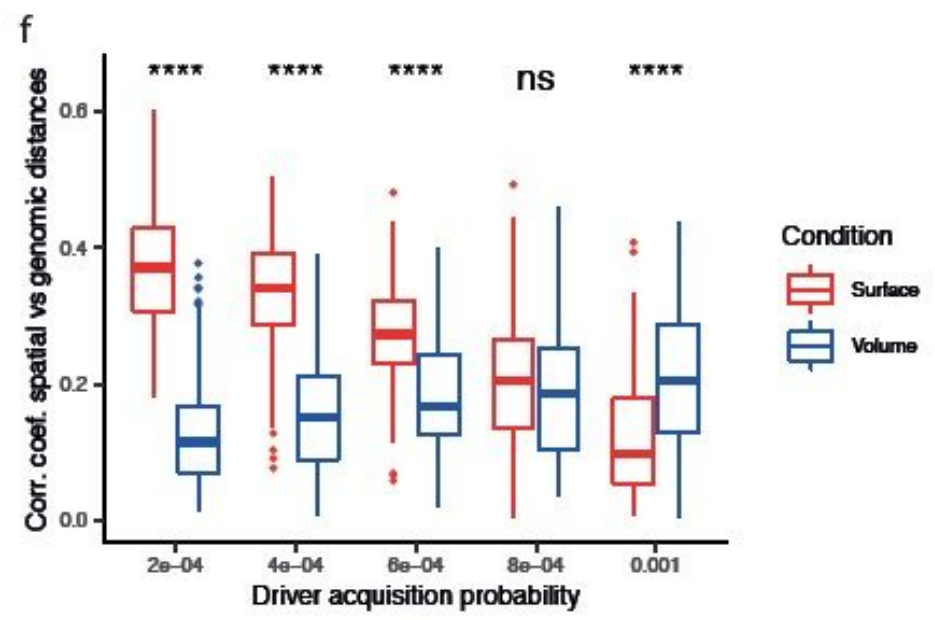

Figure 3 
Between-region genomic divergence. (a) Schematic figure and procedure for the analysis in 2D tumour regions. (b) Spatial locations of regional samples with tumour edge indicated in a representative in silico tumour under Surface Growth. (c) Between-region spatial distance against genomic distance derived from the representative in silico tumour in panel b. Overlapping data points are counted (" $n$ "). Pearson correlation (" $r$ ") and p value are indicated in the figure. (d-e) The same analysis as described in (b-c) for a representative in silico tumour with Volume Growth. (f) Pearson correlation between spatial distance and genomic distance in in silico tumours under Volume Growth and Surface Growth with varying driver acquisition probabilities. $N=100$ for each condition. Statistical annotations in (f) reflect

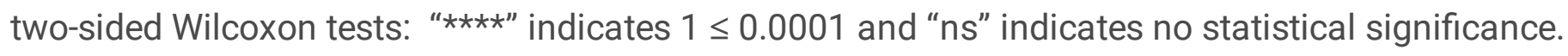


a

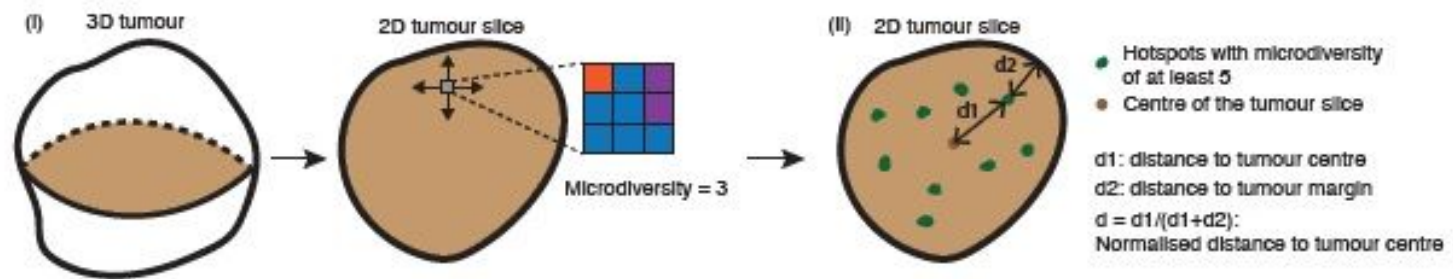

(I) Map microdlversilty within a tumour sllce by sllding a $3 \times 3$ spatial window within the tumour silce.

(ii) Evaluate the spatial pattern of microdlversity hotspots within the tumour silce.
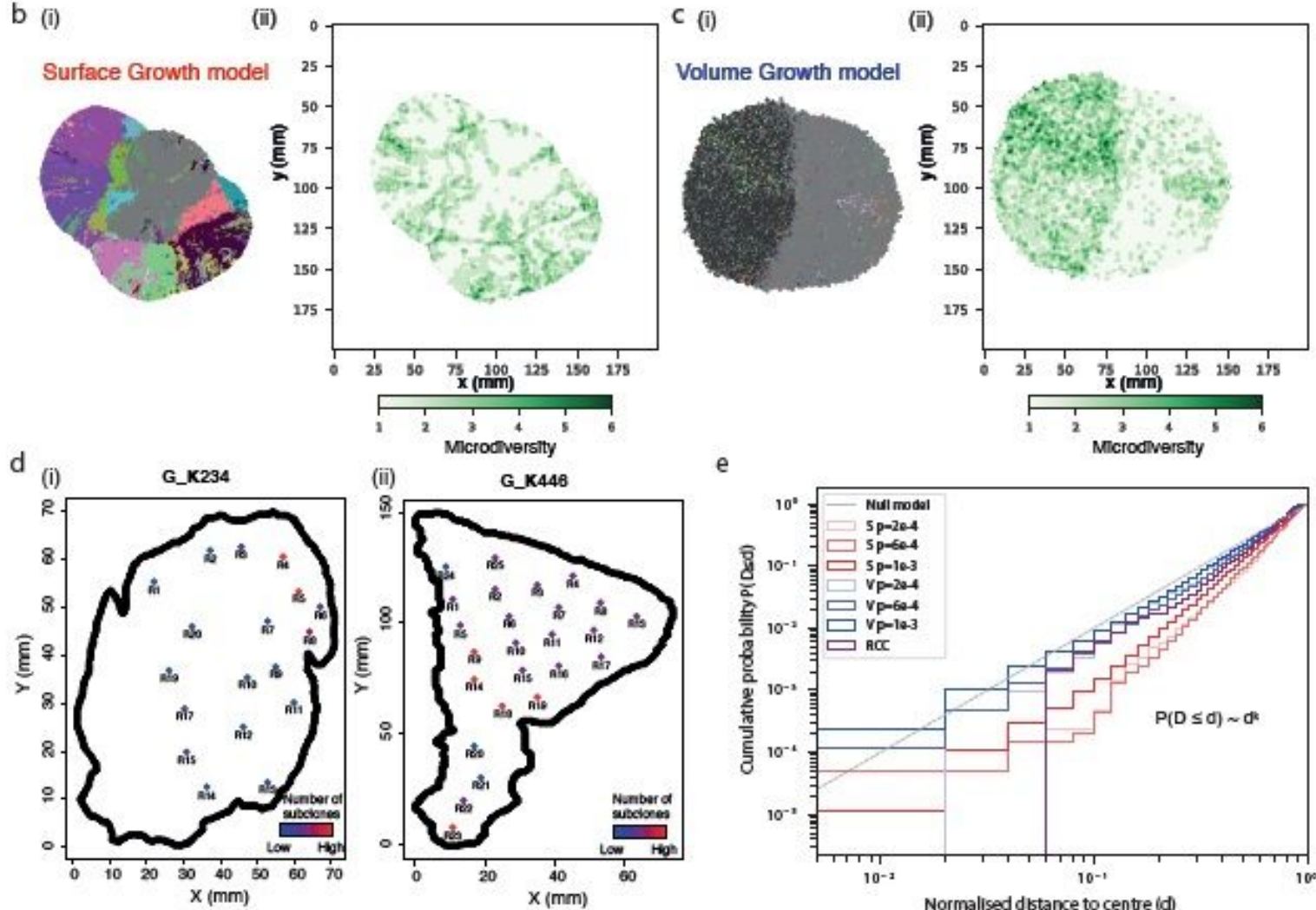

e
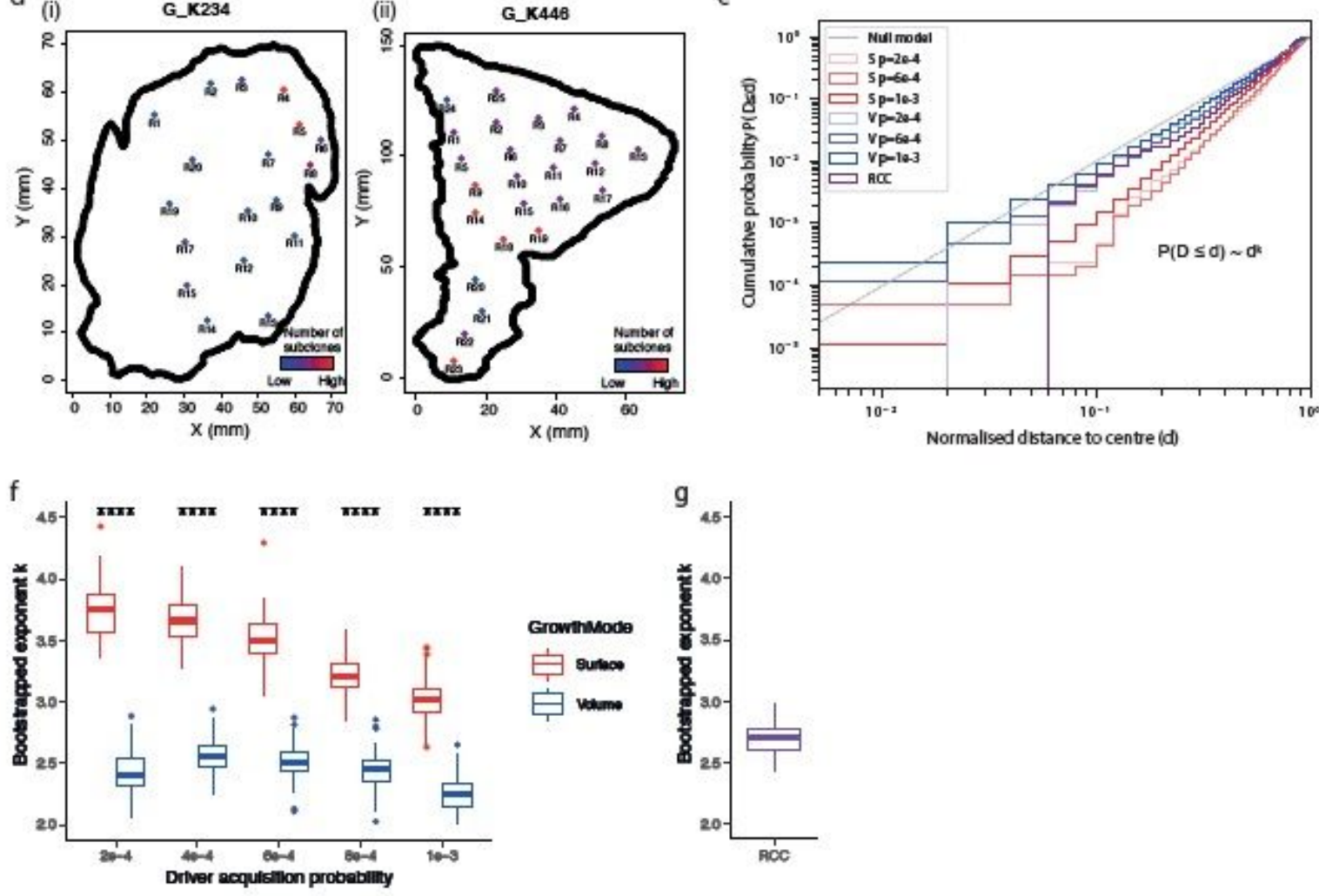

Figure 4

Spatial features of clonal diversity. (a) Schematic figure and procedure for the analysis within a 2D tumour. (b) Spatial maps of subclones (i) and microdiversity (ii) in a representative in-silico tumour under Surface Growth. (c) The same analysis as described in (b) for a representative in-silico tumour under Volume Growth. (d) Maps of regional biopsies with the number of subclones within a biopsy colour coded in two cases (G_K234 and G_K446) in the TRACERx Renal study. Hues from red to purple to blue 
reflect decreasing number of subclones. "Low" reflect zero subclones, while "High" reflects the maximum number of subclones found in any region (i.e., 4 subclones in G_K234, 3 subclones in G_K446). (e) Cumulative probability distribution, $1(2 \leq 0)$, of the normalised distance to tumour centre in in silico tumours under Surface Growth and Volume Growth and in ccRCC tumours. Three sets of in-silico tumours with different driver acquisition probabilities are shown. $\mathrm{N}=100$ for each model condition. "S" and " $V$ " in the figure reflect Surface Growth and Volume Growth, respectively. " $p=2 e-4$ " reflects a driver acquisition probability of 2e-4. 606 patient tumour (PT) regions from 54 ccRCC tumours are considered for the experimental analysis. (f) Bootstrapped power law exponent 4 , as in $1(2 \leq 0) \sim 02$, fitted to cumulative probability distribution of normalised distance to tumour centre in each of bootstrap samples. For microdiversity hotspots under each model condition, 100 bootstrap samples are generated by randomly sampling 400 hotspots with replacement. (g) Bootstrapped power law exponent 4 in ccRCC tumours. Driver acquisition probabilities used in representative simulations are: \$,\#-./\# = $2 \times 1001$ in both in-silico tumours under Surface Growth (b) and in-silico tumours under Volume Growth (c). Statistical

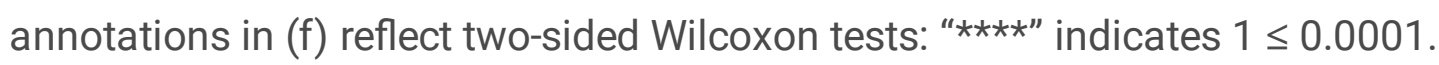


$a_{\text {(i) }}$

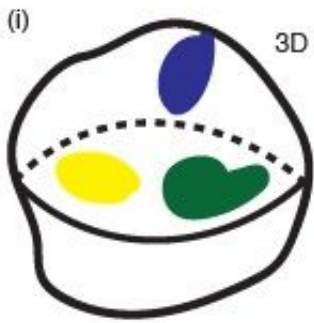

(ii) $2 \mathrm{D}$ tumour regions

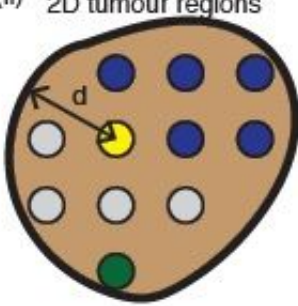

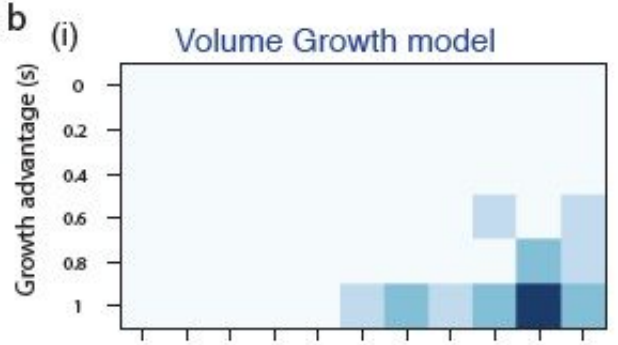

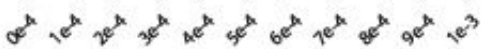

Driver acquisition probability $\left(\mathrm{p}_{\text {driver }}\right)$

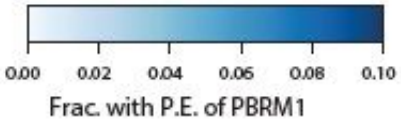

d: distance to tumour margin from events with limited clonal expansion

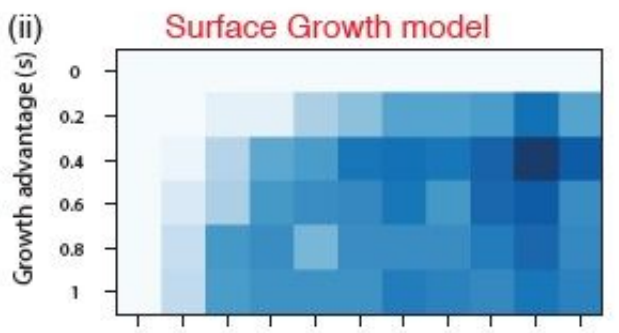

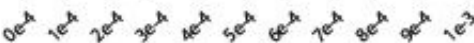

Driver acquisition probability $\left(\mathrm{p}_{\text {driver }}\right.$ )

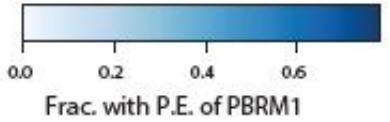

(iii)

d

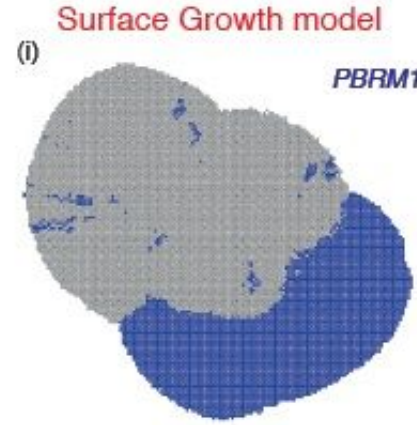

(ii)

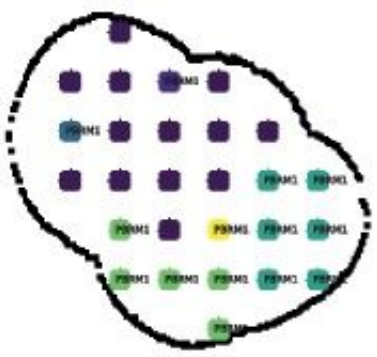

(i)

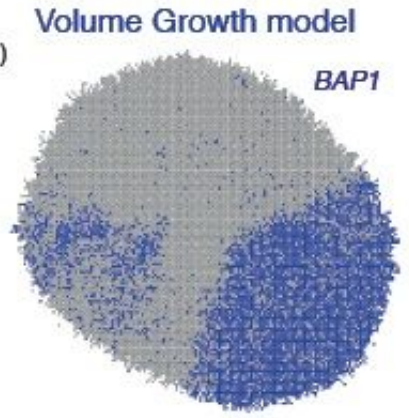

(ii)

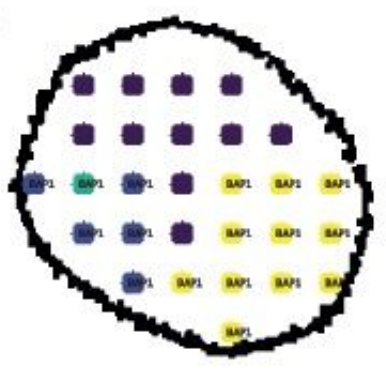

f

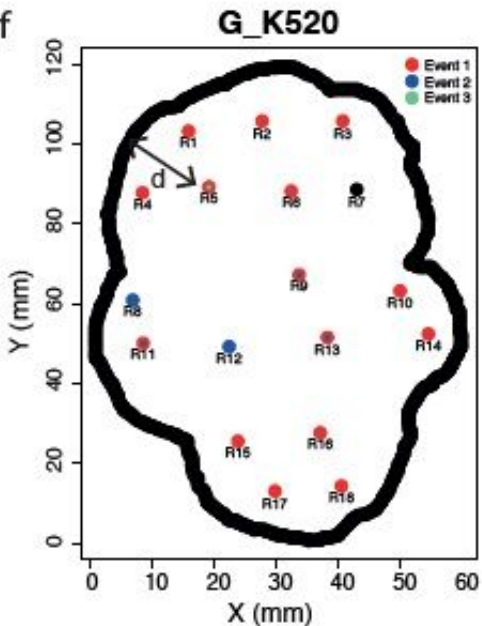

g

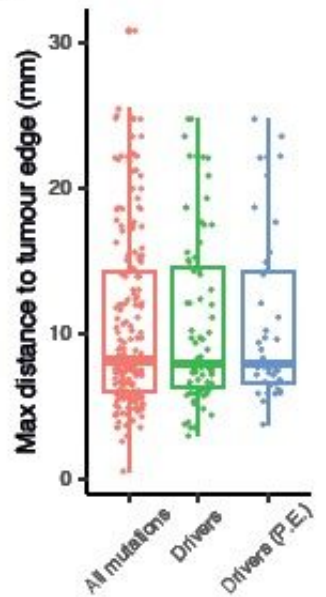

Figure 5

Frequency and spatial features of parallel evolution. (a) Schematic figure for measuring parallel evolution in the 3D tumour (i) and for analysing parallel mutational events within 2D tumour regions (ii). (b) Heatmap showing the fraction of in silico tumours that have parallel evolution in PBRM1 with respect to driver acquisition probability and growth advantage in the Volume Growth (i) and Surface Growth (ii) models. The fraction is calculated based on 50 in silico tumours per parameter condition. In silico tumours are counted, if there are two or more parallel mutational events in PBRM1 with a whole-tumour CCF of at least 0.05. (c-d) Spatial distribution of PBRM1 or BAP1 mutation (i) and its parallel events (ii) in 
a representative in silico tumour under Surface Growth (c) and Volume Growth (d), respectively. In panel (ii), different colours overlaid with "PBRM1" or "BAP1" reflect different parallel mutation events. (e) Maximum distances to the tumour edge from parallel mutations in PBRM1 or BAP1 that span up to two regions in in silico tumours under Volume Growth and Surface Growth with varying driver acquisition probabilities. $\mathrm{N}=100$ for each condition. (f) Maps of regions containing parallel mutations in PBRM1 in a representative case (G_K520) in the TRACERx Renal study. Distinct parallel mutations are coloured differently. For regions containing more than two parallel mutations, two colours are applied simultaneously. Doubleheaded arrow indicates a measurement of distance to tumour edge in this example. (g) Maximum distances from mutational events that span up to two regions in ccRCC tumours in the TRACERx Renal study. For comparison, all mutations ("All mutations"), mutations in driver genes ("Drivers"), and parallel mutations in driver genes ("Drivers (P.E.)”) are shown as separate groups. Driver acquisition probabilities used in representative simulations are: \$,\#-./\# = $2 \times 1001$ in in silico tumours under Surface Growth and \$,\#-./\# = $1 \times 100$ ! in in silico tumours under Volume Growth. Statistical annotations in (e) reflect two-sided Wilcoxon tests: " $\star \star$ " indicates $1 \leq 0.01$, “*” indicates $1 \leq 0.05$, and "ns" indicates no statistical significance. 


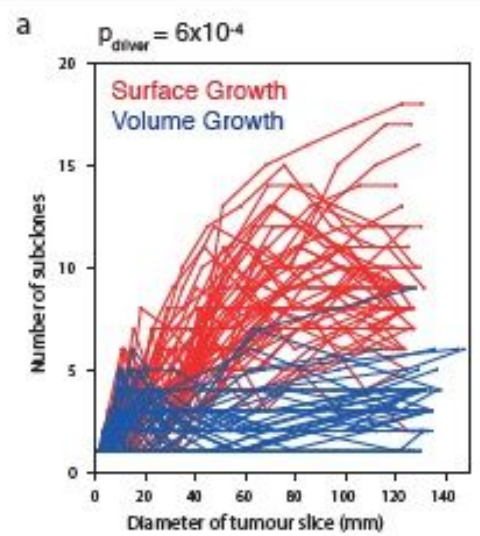

b (i) Volume Growth model

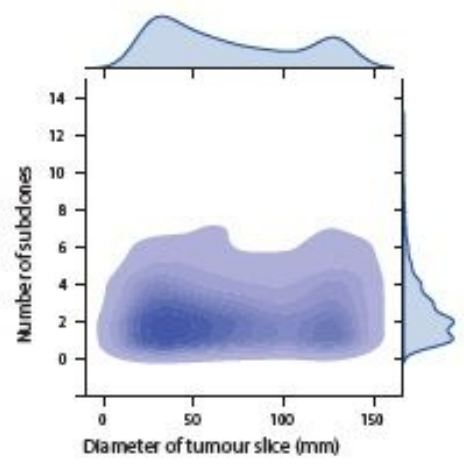

C (I)

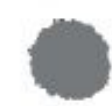

(II)

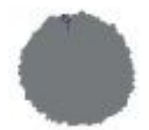

Time

d

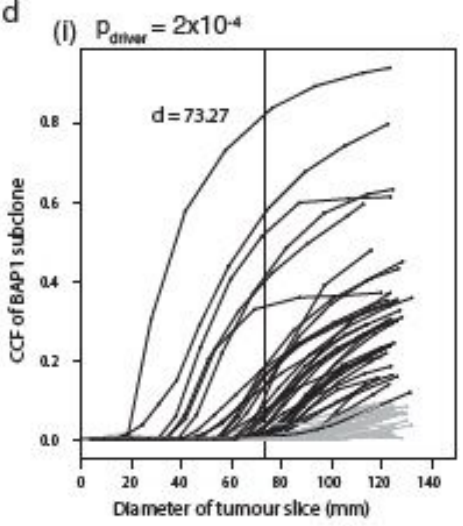

e
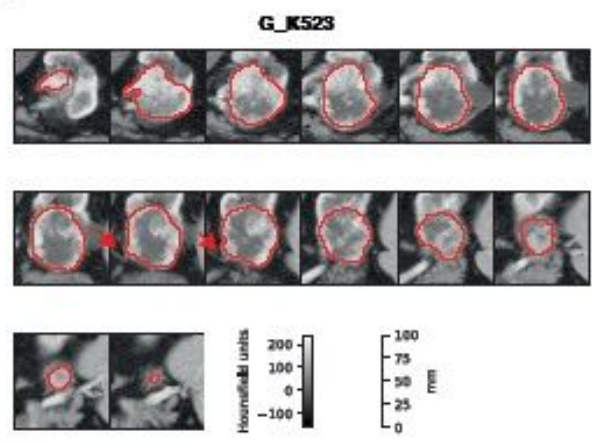

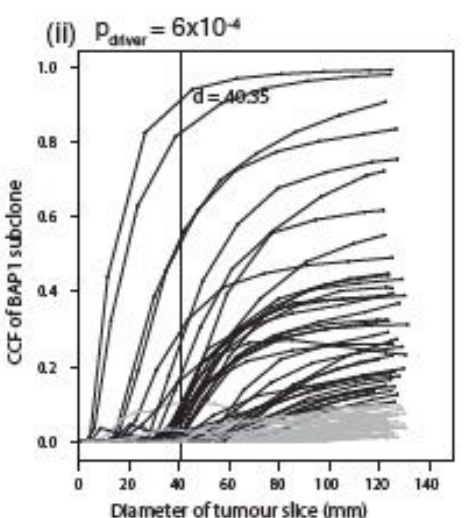

f

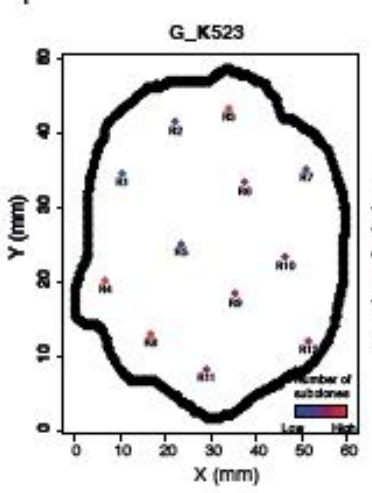

(ii) Surface Growth model

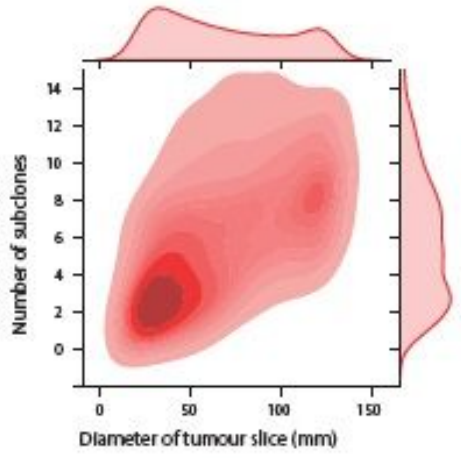

(v)
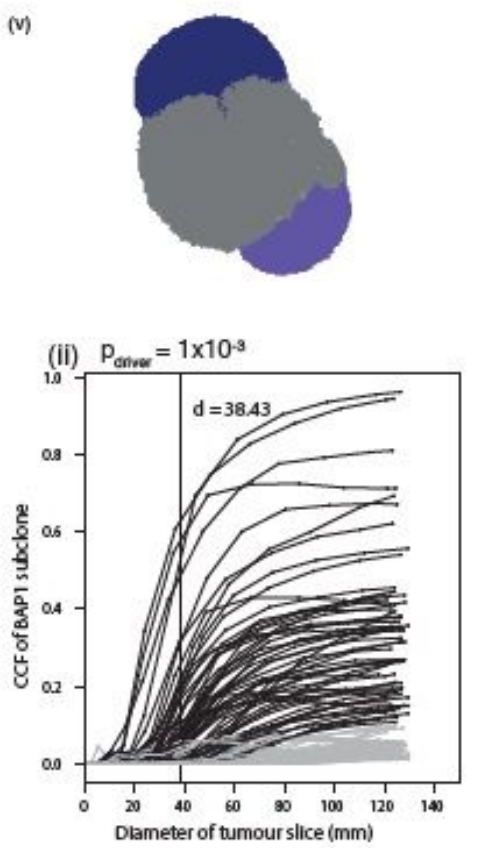

g

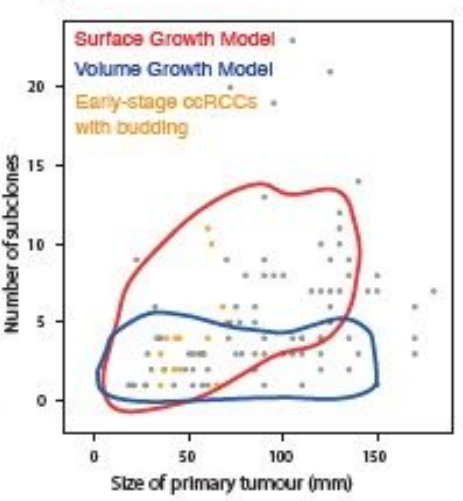

Figure 6

Early predictive indicators of evolutionary trajectories. (a) The number of subclones as a function of the diameter of a 2D tumour slice in in silico tumours under Surface Growth and under Volume Growth, respectively. $N=50$ simulations with $\$$,\#-./\# = $6 \times 1001$ are shown for each condition. (b) Kernel density estimation (KDE) with respect to the number of subclones and the diameter of a 2D tumour slice in in silico tumours under Volume Growth (i) and under Surface Growth (ii). Each KDE plot is based on 250 
simulations (50 per condition) under 5 conditions with \$, \#-/\# = $2 \times 1001,4 \times 1001,6 \times 1001,8 \times 1001,1$ $\times 100$ !. (c) The spatial patterns of parallel mutations in BAP1 over time in a representative in silico tumour under Surface Growth. The red arrow indicates a budding structure at early stage of subclonal expansion. (d) The CCFs of BAP1 clones within the 2D tumour slice as a function of the diameter of a 2D tumour slice in each of 50 in silico tumours. Only BAP1 clones with an ultimate CCF of 0.01 or higher are shown. For those with an ultimate CCF of 0.1 or higher, the diameters of tumour slices at which these clones first became detectable are recorded, with the median indicated by the dashed line. The others with an ultimate CCF of 0.1 or lower are shown in grey. (e) Axial image in the corticomedullary contrast phase of a representative case (G_K523) showing budding structure on the tumour surface (red arrow). Outlines in red were drawn giving volumetric tumour coverage by an oncologist (S.S.) and a radiologist (D.A.) (f) Maps of tumour regions with the number of subclones colour coded in a representative case (G_K523). Hues from red to purple to blue reflect decreasing number of subclones. "Low" reflect zero subclones, while "High" reflects the maximum number of subclones found in any region (i.e., 4 subclones in G_K523). (g) The number of subclones as a function of ultimate tumour size in the TRACERx Renal study, overlaid with kernel density estimation based on imulated data. Tumours with a size smaller than $7 \mathrm{~cm}$ and with radiologically evident budding structures on the tumour surface are highlighted (orange). Contours reflect $90 \%$ probability density based on in silico tumours under Surface Growth (red) and under Volume Growth (blue), respective in Figure 6b.

\section{Supplementary Files}

This is a list of supplementary files associated with this preprint. Click to download.

- NEESubmissionSupplementallnfotextfigurescombinedXF.pdf 